\title{
LETTER REPORT
}

Nuclear Science and Technology Division (94)

Nuclear Fuel Cycle Analysis Section

Project Title:

ORNL Support for Yucca Mountain Project

Subject of Document: Fiscal Year 2001 Status Report for the Investigation of Reactivity Effects Due to Perturbations in Cross-Section Temperature

Type of Document: $\quad$ Letter Report

Author(s):

M. E. Dunn

Date Published:

September 2001

Responsible YMP

D. A. Thomas (702) 295-4507

Individual:

Prepared for

Bechtel SAIC Company, LLC

$\mathrm{M} \& \mathrm{O}$ Contractor

DOE Office of Civilian Radioactive Waste Management

Prepared by the

OAK RIDGE NATIONAL LABORATORY

P. O. Box 2008

Oak Ridge, Tennessee 37831-6370

managed by

UT-BATTELLE, LLC

for the

U. S. DEPARTMENT OF ENERGY

under contract DE-AC05-00OR22725

\section{LETTER REPORT}




\section{CONTENTS}

$\underline{\text { Page }}$

LIST OF FIGURES $\ldots \ldots \ldots \ldots \ldots \ldots \ldots \ldots \ldots \ldots \ldots \ldots \ldots \ldots \ldots \ldots \ldots$ iii

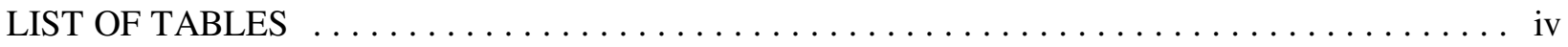

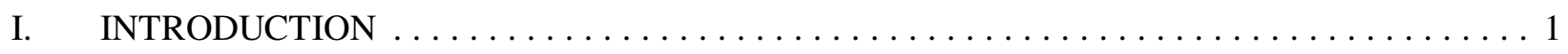

II. $\quad$ TASK 1: CROSS-SECTION LIBRARY GENERATION $\ldots \ldots \ldots \ldots \ldots \ldots \ldots \ldots \ldots \ldots$.

III. TASK 2: 1-D DISCRETE ORDINATES SENSITIVITY CALCULATIONS . . . . . . . . 5

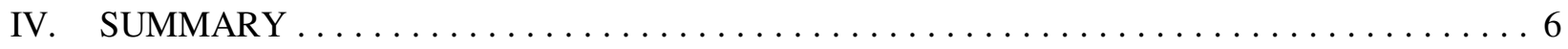

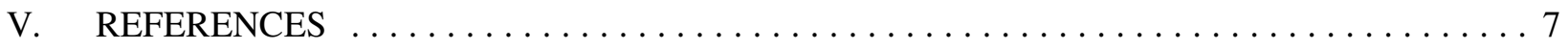

APPENDIX A: $\quad$ SUMMARY OF ENDF/B-VI EVALUATIONS PROCESSED WITH AMPX-2000 . 8

APPENDIX B: CONTENTS OF 238-GROUP ENDF/B-VI LIBRARY GENERATED

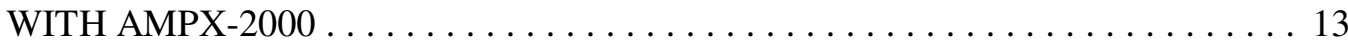

APPENDIX C: EXAMPLE OUTPUT OF PROCESSING CRC BENCHMARKS WITH

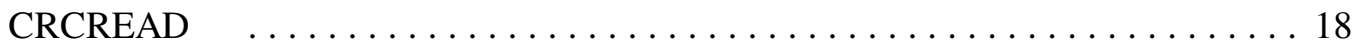




\section{LIST OF FIGURES}

$\underline{\text { Figure }}$

$\underline{\text { Page }}$

1. AMPX flow diagram for processing a nonthermal cross-section evaluation $\ldots \ldots \ldots \ldots \ldots$ 


\section{LIST OF TABLES}

$\underline{\text { Table }}$

$\underline{\text { Page }}$

1. Work scope for temperature effects study $\ldots \ldots \ldots \ldots \ldots \ldots \ldots \ldots \ldots \ldots \ldots \ldots \ldots \ldots$

2. AMPX flow diagram for processing nonthermal cross-section evaluations $\ldots \ldots \ldots \ldots \ldots \ldots$

3. Functional description of AMPX modules used to process nonthermal cross-section evaluations _...4 


\section{INTRODUCTION}

The work in this report has been performed in support of the Yucca Mountain Project (YMP), and the objective of this work is to determine the impact on predictions in system multiplication due to perturbations in cross-section temperature. Moreover, the study is intended to investigate the impact of cross-section perturbations on LWR spent fuel isotopics at various levels of burnup. This letter report provides the current status of the temperature-effects work and documents the work that has been completed during the 2001 fiscal year.

The work scope for the temperature-effects study is outlined in Table 1. The tasks that are presented in Table 1 are organized in a sequential fashion. As a result, the completion of each task is dependent upon the completion of the preceding tasks. The majority of effort during the fiscal year has been devoted to completion of the first task. In addition, some effort has been devoted to the second task. Therefore, this report summarizes the efforts related to the completion of Tasks 1 and 2.

Table 1. Work scope for temperature effects study

\begin{tabular}{|c|c|}
\hline Task & Description \\
\hline 1 & $\begin{array}{l}\text { Generate an ENDF/B-V } L^{1} \text { multigroup library for } \mathrm{SCALE}^{2} \text { using the AMPX-200 } \mathrm{L}^{3} \text { cross-section } \\
\text { processing system. }\end{array}$ \\
\hline 2 & $\begin{array}{l}\text { Use the 1-D discrete ordinates code XSDRNPM }{ }^{2} \text { to calculate changes in reactivity due to } \\
\text { temperature perturbations for each isotope/nuclide over a range of temperature (to be } \\
\text { determined). The XSDRNPM calculations will be performed with the ENDF/B-VI multigroup } \\
\text { library developed in Task } 1 \text {. The isotopes/nuclides that exhibit significant changes in reactivity } \\
\text { will be considered the "relevant isotopes." Note that the XSDRNPM calculations will consist of } \\
\text { infinite lattice models of LWR assemblies at different levels of burnup. }\end{array}$ \\
\hline 3 & $\begin{array}{l}\text { Use the criticality safety code KENO V.a }{ }^{1} \text { with the ENDF/B-VI library from Task } 1 \text { to evaluate } \\
\text { reactor and laboratory criticals. Specifically, the criticality calculations for the criticals will } \\
\text { examine the temperature effects on the relevant isotopes that are identified in Task } 2 \text {. }\end{array}$ \\
\hline 4 & $\begin{array}{l}\text { Use } \mathrm{NJOY}^{4} \text { to generate } \mathrm{MCNP}^{5} \text { temperature-dependent continuous-energy cross section for } \\
\text { relevant isotopes identified in Task } 2 \text {. }\end{array}$ \\
\hline 5 & Use MCNP with cross sections from Task 4 to "spot check" KENO calculations from Task 3. \\
\hline 6 & Document temperature effects work in ORNL/TM document. \\
\hline
\end{tabular}




\section{TASK 1: CROSS-SECTION LIBRARY GENERATION}

In the United States, the Evaluated Nuclear Data File (ENDF) system is a repository for evaluated nuclear data that are obtained from cross-section measurements. The objective of this work is to use the most up-to-date cross-section information that is available, and the latest cross-section information that is available to the nuclear analyst is ENDF/B-VI. Because of the vast amount of nuclear data that is available in a crosssection evaluation, radiation transport codes do not access the ENDF/B data directly. As a result, a crosssection processing code system must be used to access the ENDF data and generate cross-section libraries for radiation transport codes. At the Oak Ridge National Laboratory (ORNL), the AMPX code system is used to process ENDF evaluations and generate cross-section libraries. 3 Moreover, the SCALE (Standardized Computer Analyses for Licensing Evaluations) system, which is also developed and maintained at ORNL, is used throughout the world to investigate technical issues related to the nuclear fuel cycle.2 The objective of the first task is to use AMPX to process the latest ENDF cross-section data (ENDF/B-VI) and generate a multigroup library that can be used in the subsequent temperature-effects study.

For the purposes of this task, the latest AMPX code system (i.e., AMPX-2000) which is prototypic in nature is being used to generate a 238-group cross-section library for use in the SCALE system. The energy-group structure for the ENDF/B-VI library is identical to the ENDF/B-V 238-group structure ${ }^{2}$ that is currently available in SCALE.

In ENDF/B-VI, there are currently cross-section evaluations for 325 different isotopes/nuclides. In addition, there are thermal evaluations [i.e., $S(\alpha, \beta)$ scattering kernels] for 15 different materials (e.g., hydrogen in water, etc.). Each cross-section evaluation contains a variety of data that are used to model the radiation transport through each material. As a result, the cross-section processing requirements vary between isotopes. Moreover, the different types of data that are available in a cross-section evaluation require different AMPX modules to process the data. Consequently, an AMPX input file must be generated for each individual isotope/nuclide.

Although there are cross-section evaluations for more than 300 different isotopes/nuclides, there may be multiple ENDF/B-VI evaluations for each different isotope/nuclide. For the purposes of this task, a series of FORTRAN programs were developed to scan the entire ENDF/B-VI database and compile a concise directory information for each isotope/nuclide. Subsequently, an additional FORTRAN program (MAKEINPUTS) was developed to read the cross-section database and select the most recent cross-section evaluation for each isotope/nuclide. Because the cross-section processing requirements vary between isotopes, MAKEINPUTS was also used to generate AMPX input files for the 325 isotopes/nuclides and 15 thermal scattering evaluations. A brief summary of each material that has been processed with AMPX is presented in the Appendix A. Note that the series of FORTRAN programs that were developed as part of this task were used to generate the listing in Appendix A.

As indicated previously, the type of data that are present in a cross-section evaluation dictates the specific procedures that must be used to process the evaluation (e.g., the evaluation may have resolvedresonance parameters specified with the Reich-Moore formalism, the evaluation may not have resonance information, etc.). Because the AMPX code system is very modular in nature, each AMPX module performs a specific cross-section task by reaction type and format specification. This information varies for the different nuclide evaluations. As a result, all modules are not executed for each cross-section evaluation. As part of the cross-section library production effort, MAKEINPUTS selects the appropriate AMPX modules needed to process each nuclide. 
In order to illustrate the logic for processing a nonthermal evaluation, Figure 1 provides a flow diagram for selecting the appropriate AMPX modules to process an evaluation. MAKEINPUTS performs the decisionmaking steps as indicated in Figure 1 for a nonthermal cross-section evaluation. The definitions for each symbol are provided in Table 2, and the functional description of each AMPX module is provided in Table 3. Although different AMPX modules may be executed for different cross-section evaluations, the final product of the processing task is an AMPX-formatted master file for each material which is denoted by the letter "A" in Figure 1. The flow logic in Figure 1 has been applied to each of the 325 nonthermal evaluations in ENDF/B-VI.

After processing all of the cross-section evaluations, the AJAX module in AMPX was used to combine the master-formatted crosssections into a single 238-group ENDF/B-VI library. The contents of the multigroup library that has been generated in this task is provided in Appendix B. Although the 238-group ENDF/B-VI library has been generated, efforts are currently in progress to test the library for accuracy. Once the library is tested, the subsequent tasks that are provided in Table 1 of Section 1 can be completed.

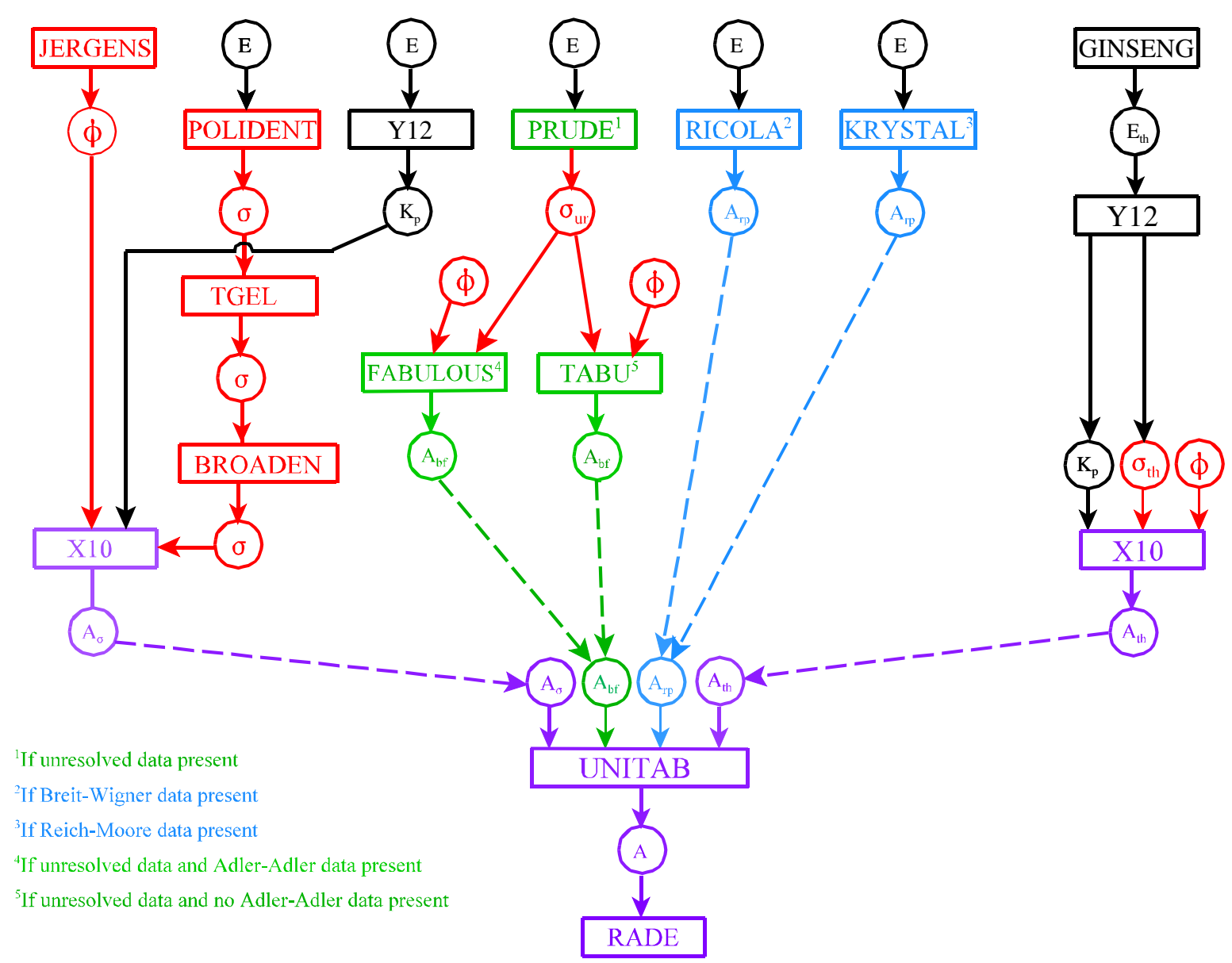

Fig. 1. AMPX flow diagram for processing a nonthermal cross-section evaluation 
Table 2. AMPX flow diagram for processing nonthermal cross-section evaluations

\begin{tabular}{ll}
\hline Task & \multicolumn{1}{c}{ Description } \\
\hline $\mathrm{A}$ & AMPX master-formatted cross-section library \\
$\mathrm{A}_{\mathrm{bf}}$ & AMPX master-formatted cross-section library containing only Bondarenko factors \\
$\mathrm{A}_{\mathrm{rp}}$ & AMPX master-formatted cross-section library containing only resonance parameters \\
$\mathrm{A}_{\mathrm{th}}$ & AMPX master-formatted cross-section library containing only thermal cross-sections \\
$\mathrm{A}_{\sigma}$ & AMPX master-formatted cross-section library containing only 1-D and 2-D cross-sections \\
$\mathrm{E}$ & ENDF evaluation \\
$\mathrm{E}_{\mathrm{th}}$ & Free-gas thermal data in ENDF format \\
$\mathrm{K}_{\mathrm{p}}$ & Collision kinematics (i.e., secondary energy and angle) data for each reaction that emits \\
& secondary neutron(s) \\
$\phi$ & Weighting spectrum \\
$\sigma$ & Continuous-energy cross sections \\
$\sigma_{\mathrm{th}}$ & Continuous-energy cross sections at thermal energies (i.e., $<5$ eV) \\
$\sigma_{\mathrm{ur}}$ & Continuous-energy cross sections in the unresolved-resonance region \\
\hline
\end{tabular}

Table 3. Functional description of AMPX modules used to process nonthermal cross-section evaluations

\begin{tabular}{ll}
\hline \multicolumn{1}{c}{ Module } & \\
\hline BROADEN & Doppler broadens continuous-energy cross-section data \\
FABULOUS & Generates Bondarenko self-shielding factors for the entire resonance region \\
GINSENG & Generates free-gas collision data at thermal energies for nonthermal evaluations \\
JERGENS & Calculates a weighting spectrum for use in generating a multigroup library \\
KRYSTAL & Converts Reich-Moore resonance parameters to the multipole formalism \\
POLIDENT & Calculates continuous-energy cross-sections at 0 K \\
PRUDE & $\begin{array}{l}\text { Reads unresolved resonance parameters and constructs continuous-energy cross } \\
\text { sections in the unresolved-resonance region }\end{array}$ \\
RADE & Performs consistency checks on an AMPX master library \\
RICOLA & $\begin{array}{l}\text { Extracts single- and multi-level Breit-Wigner resonance parameters from an ENDF } \\
\text { evaluation and writes to an AMPX master library }\end{array}$ \\
TGEL & Ensures that the sum of the partial reactions equals the total cross section \\
UNITAB & $\begin{array}{l}\text { Assembles a complete AMPX master library } \\
\text { X10 }\end{array}$ \\
Performs integrations to calculate group-averaged cross sections and scattering matrices \\
Y12
\end{tabular}




\section{TASK 2: 1-D DISCRETE ORDINATES SENSITIVITY CALCULATIONS}

The objective of the second task is to develop 1-D discrete ordinates (DO) models for LWR spent fuel at different levels of burnup. The XSDRNPM module, which is in the SCALE package, will be used to perform the 1-D DO calculations using the ENDF/B-VI multigroup library developed in Task 1. As noted in Section I, the majority of effort during the fiscal year has been devoted to the library production task that is discussed in Section II. However, some effort has been devoted to the development of XSDRNPM models for the temperature perturbation calculations.

One of the essential tasks for the XSDRNPM model development activities is the identification of isotopic-concentration specifications for the various isotopes that are to be included in the calculational models. In an effort to identify the appropriate isotopic concentrations, a FORTRAN program (CRCREAD) has been developed to read the MCNP input files for the commercial reactor criticals (CRC) benchmark models and extract information that will be used to create the XSDRNPM models. The YMP has designated 130 different isotopes, which are documented in Appendix C, that are of interest for the temperature-effects study. CRCREAD has the capability to search a CRC benchmark model for the 130 different isotopes and determine which isotopes are in the model. If one of the designated isotopes is present, the code determines the physical location of each isotope (i.e., in the fuel, in the structure outside of the fuel region or in both locations). In addition, CRCREAD determines the weight fraction of the isotope in the respective material location. As work continues on Task 2, CRCREAD will be expanded to calculate the atomic-number densities for isotopes that will be used in the XSDRNPM models. In an effort to demonstrate the capabilities of CRCREAD, the benchmark case cr3i12a was processed with CRCREAD, and a summary of the output is also provided in Appendix C. 


\section{SUMMARY}

The objective of the current work is to calculate changes in system multiplication due to perturbations in cross-section temperature. Moreover, the study is intended to investigate cross-section perturbations using ENDF/B-VI data which are the most up-to-date cross-section information currently available in the United States. The purpose of this letter report is to document the work that has been completed during the 2001 fiscal year. In addition, the report provides the current status of the temperature-effects study.

During the fiscal year, the majority of effort has focused on the development of a 238-group ENDF/B-VI library using the AMPX cross-section processing system. At this point, $340 \mathrm{ENDF} / \mathrm{B}-\mathrm{VI}$ evaluations have been processed (i.e., 15 thermal and 325 nonthermal evaluations), and a prototypic 238-group library has been developed. Efforts are currently in progress to test the cross-section library for accuracy. Once the library is determined to be suitable for radiation transport calculations, the library will be used to perform reactivity calculations to assess the impact of cross-section temperature perturbations.

Regarding the temperature perturbation studies, a FORTRAN program (CRCREAD) has been developed to read the CRC benchmark models (i.e., MCNP input files) and search for the 130 different isotopes that have been designated by the YMP for further study. Currently, CRCREAD determines if an isotope is present in the $\mathrm{CRC}$ benchmark model and determines the physical location of the isotope within the model. In addition, CRCREAD determines the weight fraction of the isotope in a material within the MCNP model. In the future, CRCREAD will be used as a tool to aide in the development of 1-D XSDRNPM input models that will be used in the temperature-effects calculations.

The work that has been completed during the 2001 fiscal year has provided the necessary foundation for completing the temperature effects study. During the 2002 fiscal year, the 238-group ENDF/B-VI library that was developed in Task 1 will be tested for accuracy. Subsequently, XSDRNPM models of LWR spent fuel assemblies will be developed, and the new 238-group library will be used with XSDRNPM to calculate reactivity changes due to cross-section temperature perturbations for the LWR models. The XSDRNPM models will be used to identify the important or relevant isotopes requiring further study. Moreover, these relevant isotopes will be evaluated by using KENO V.a with the new 238-group library to calculate specific reactor and laboratory criticals. The 3-D KENO V.a calculations will be used to examine reactivity effects

due to temperature changes of the relevant isotopes. Finally, MCNP will be used to "spot check" the KENO V.a calculations in order to verify the results of the temperature-effects study. 


\section{REFERENCES}

1. "ENDF-102 Data Formats and Procedures for the Evaluated Nuclear Data File ENDF-6," BNL-NCS44945, Rev. 10/91 (ENDF/B-VI), Brookhaven National Laboratory, October 1991.

2. SCALE: A Modular Code System for Performing Standardized Computer Analyses for Licensing Evaluation, NUREG/CR-0200, Rev. 6 (ORNL/NUREG/CSD-2/R6), Vols. I, II, and III, May 2000. Available from Radiation Safety Information Computational Center at Oak Ridge National Laboratory as CCC-545.

3. N. M. Greene and M. E. Dunn, AMPX-2000: A Modular Code System for Processing ENDF/B Evaluations, NUREG/CR-6659 (ORNL/TM-1999/265), September 2001, Draft for NRC review.

4. R. E. MacFarlane and D. W. Muir, "NJOY99.0 Code System for Producing Pointwise and Multigroup Neutron and Photon Cross Sections from ENDF/B Data," PSR-480/NJOY99.0, Los Alamos National Laboratory, March 2000.

5. "MCNP 4C: Monte Carlo N-Particle Transport Code System," Los Alamos National Laboratory LA-3709-M (April 2000). 


\section{APPENDIX A SUMMARY OF ENDF/B-VI EVALUATIONS PROCESSED WITH AMPX-2000}

The following is a summary of the 340 ENDF/B-VI evaluations that have been processed with AMPX. For each evaluation, the symbol and 4-digit ENDF material identifier are provided for each isotope along with the appropriate ENDF tape information. The tape information provides the ENDF mod number, release number, ZA number and evaluation date (evdt) for the cross-section evaluation. In addition, the name of the evaluator along with the organization that performed the evaluation is provided (e.g., LANL, ORNL, etc.). Following the organization information is the ENDF filename (i.e., source), distribution date and rev number.

The remaining information for each evaluation describes the neutron resolved-resonance formalism that is used in File 2 of the ENDF evaluation. The possible resonance formalisms are as follows:

$\begin{array}{ll}\text { slbw } & \text { Single-level Breit-Wigner } \\ \text { mlbw } & \text { Multi-level Breit-Wigner } \\ \text { rm } & \text { Reich-Moore } \\ \text { aa } & \text { Adler-Adler } \\ \text { grm } & \text { General R Matrix } \\ \text { hrf } & \text { Hybrid R Function }\end{array}$

Following the resonance information is the File 3 data that are provided for each ENDF evaluation. The File 3 information provides the neutron induced reactions that are available for the specific isotope. If the evaluation is a thermal evaluation [i.e., $\mathrm{S}(\alpha, \beta)$ data], the evaluation has an ENDF File 7 (i.e., $\mathrm{MF}=7$ ).

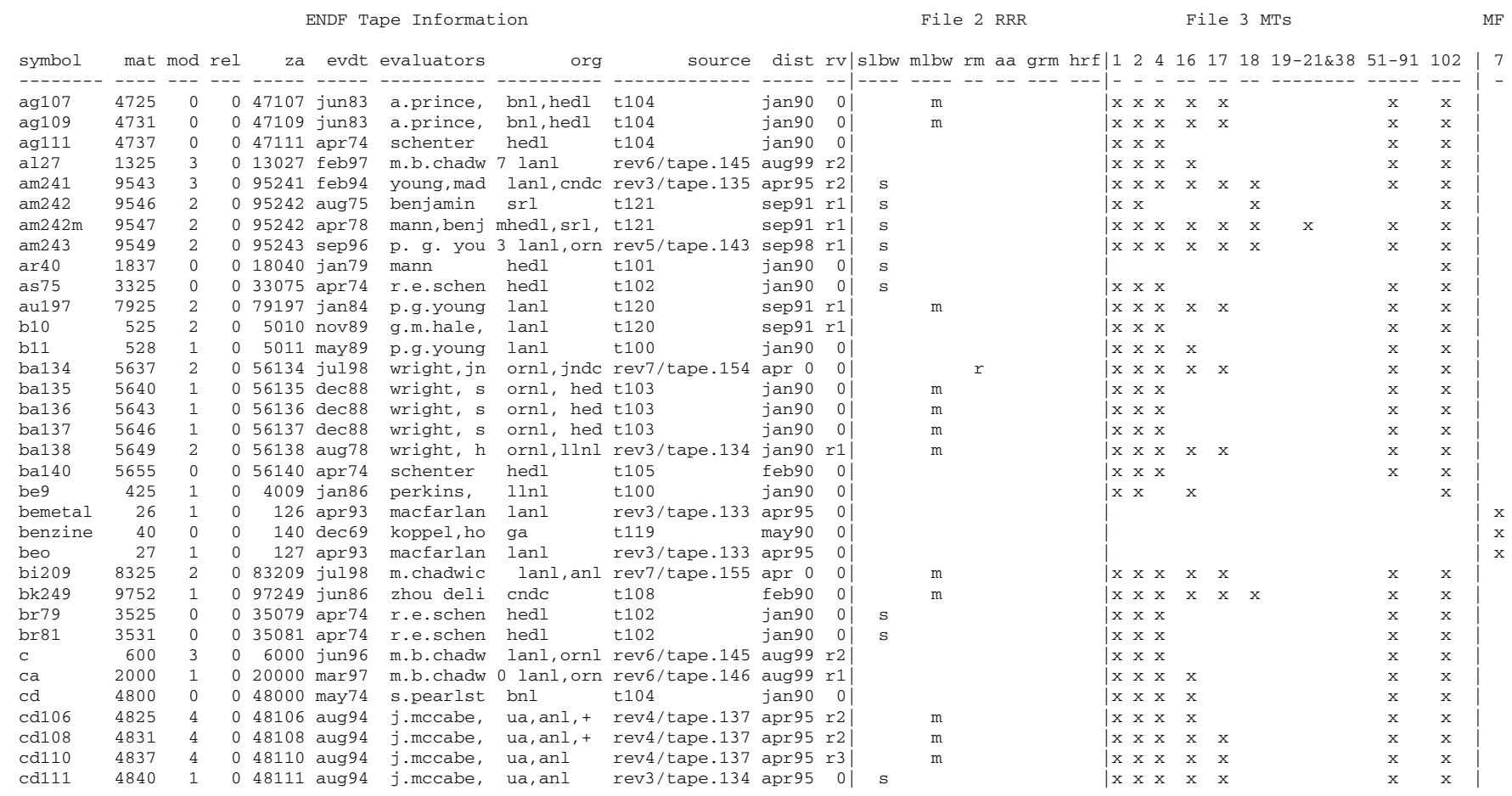




\begin{tabular}{|c|c|c|c|c|c|c|c|c|c|c|c|c|}
\hline symbol & mat & mod & $\begin{array}{l}\text { rel } \\
---\end{array}$ & $\begin{array}{r}z a \\
----\end{array}$ & $\begin{array}{r}\text { evdt } \\
-----\end{array}$ & evaluators & $\begin{array}{r}\text { org } \\
-------\end{array}$ & $\begin{array}{r}\text { source } \\
-------1\end{array}$ & dist & $\begin{array}{l}r v \mid \\
--1\end{array}$ & $\begin{array}{ll}1 \mathrm{bw} \\
---\end{array}$ & $\begin{array}{l}\text { mlbw } \\
----\end{array}$ \\
\hline $\operatorname{cd} 112$ & 4843 & 4 & 0 & 48112 & $\operatorname{aug} 94$ & j.mccabe, & ua, anl, + & rev4/tape.137 & apr95 & r2 & & $\mathrm{m}$ \\
\hline $\operatorname{cd} 113$ & 4846 & 1 & 0 & 48113 & aug94 & j.mccabe, & ua, anl & rev3/tape.134 & apr95 & 0 & $\mathrm{~s}$ & \\
\hline cd114 & 4849 & 4 & 0 & 48114 & $\operatorname{aug} 94$ & j.mccabe, & ua, anl, + & rev4/tape.137 & apr95 & r2 & & $\mathrm{m}$ \\
\hline $\mathrm{cd} 115 \mathrm{~m}$ & 4853 & 0 & 0 & 48115 & apr74 & schenter & mhedl & t104 & jan90 & 0 & & \\
\hline cd116 & 4855 & 4 & 0 & 48116 & aug94 & j.mccabe, & ua, anl, + & rev4/tape.137 & apr95 & r2 & & $\mathrm{m}$ \\
\hline ce140 & 5837 & 0 & 0 & 58140 & apr74 & r.e.schen & hedl & t105 & feb90 & 0 & & \\
\hline ce141 & 5840 & 0 & 0 & 58141 & apr74 & schenter & hedl & t105 & feb90 & 0 & & \\
\hline ce142 & 5843 & 0 & 0 & 58142 & apr74 & r.e.schen & hedl & t105 & feb90 & $0 \mid$ & & \\
\hline ce143 & 5846 & 0 & 0 & 58143 & apr74 & schenter & hedl & t105 & feb90 & $0 \mid$ & & \\
\hline ce144 & 5849 & 0 & 0 & 58144 & apr74 & schenter & hedl & t105 & feb90 & 0 & & \\
\hline $\operatorname{cf} 249$ & 9852 & 1 & 0 & 98249 & apr89 & zhou deli & cnde & t108 & feb90 & $0 \mid$ & & $\mathrm{m}$ \\
\hline $\operatorname{cf} 250$ & 9855 & 2 & 0 & 98250 & jul76 & kinsey-as & bnl, srl, + & rev2/tape.129 & jun93 & r1| & $\mathrm{s}$ & \\
\hline $\operatorname{cf} 251$ & 9858 & 2 & 0 & 98251 & jul76 & kinsey-as & bnl, srl, + & rev2/tape.129 & jun93 & r1 & $\mathrm{s}$ & \\
\hline $\operatorname{cf} 252$ & 9861 & 3 & 0 & 98252 & jul76 & kinsey-as & bnl, srl, + & rev2/tape.129 & jun93 & r2 & $\mathrm{s}$ & \\
\hline $\operatorname{cf} 253$ & 9864 & 2 & 0 & 98253 & $\operatorname{dec} 75$ & benjamin & $\operatorname{srl}$ & rev2/tape.129 & jun93 & r1 & $\mathrm{s}$ & \\
\hline cl & 1700 & 0 & 0 & 17000 & feb67 & m.s.allen & gga & t101 & jan90 & 0 & & \\
\hline $\mathrm{cm} 241$ & 9628 & 0 & 0 & 96241 & apr78 & mann and & hedl & t110 & feb90 & $0 \mid$ & & \\
\hline $\mathrm{cm} 242$ & 9631 & 1 & 0 & 96242 & apr78 & mann, benj & hedl,srl, & t109 & feb90 & $0 \mid$ & $\mathrm{s}$ & \\
\hline $\mathrm{cm} 243$ & 9634 & 1 & 0 & 96243 & jul95 & v.maslov, & minsk & rev7/tape.155 & apr 0 & 0 & $\mathrm{~s}$ & \\
\hline $\mathrm{cm} 244$ & 9637 & 0 & 0 & 96244 & $\operatorname{apr} 78$ & mann, benj & hedl, srl, & t110 & feb90 & 0 & $\mathrm{~s}$ & \\
\hline $\mathrm{cm} 245$ & 9640 & 3 & 0 & 96245 & nov95 & v.maslov & minsk & rev7/tape.155 & apr 0 & $0 \mid$ & $\mathrm{s}$ & \\
\hline $\mathrm{cm} 246$ & 9643 & 3 & 0 & 96246 & nov95 & v.maslov & minsk & rev7/tape.155 & apr 0 & $0 \mid$ & & $\mathrm{m}$ \\
\hline $\mathrm{cm} 247$ & 9646 & 2 & 0 & 96247 & jul76 & kinsey-as & bnl,srl, + & rev2/tape.129 & jun93 & $r 1$ & $\mathrm{~s}$ & \\
\hline $\mathrm{cm} 248$ & 9649 & 0 & 0 & 96248 & apr78 & mann, benj & hedl,srl, & t110 & feb90 & 01 & $\mathrm{~s}$ & \\
\hline $\operatorname{co5} 9$ & 2725 & 2 & 0 & 27059 & jul89 & a.smith+, & anl, ornl & rev2/tape.129 & jun93 & r1 & & \\
\hline $\operatorname{cr} 50$ & 2425 & 4 & 0 & 24050 & oct97 & s.chiba,m & 0 lanl, orn & rev6/tape. 147 & aug99 & $r 3$ & & \\
\hline $\operatorname{cr} 52$ & 2431 & 3 & 0 & 24052 & oct97 & s.chiba, m & 2 lanl, orn & rev6/tape. 147 & aug99 & r2 & & \\
\hline $\operatorname{cr} 53$ & 2434 & 3 & 0 & 24053 & oct97 & s.chiba, m & 3 lanl, orn & rev6/tape.147 & aug99 & r2 & & \\
\hline $\operatorname{cr} 54$ & 2437 & 4 & 0 & 24054 & oct97 & s.chiba, m & 4 lanl, orn & rev6/tape.147 & aug99 & r3 & & \\
\hline $\operatorname{cs} 133$ & 5525 & 1 & 0 & 55133 & aug99 & s.y.oh,s. & bnl, kaeri & rev7/tape.154 & apr 0 & 0 & & $\mathrm{~m}$ \\
\hline $\operatorname{cs} 134$ & 5528 & 2 & 0 & 55134 & aug98 & wright, s & ornl, hedl & rev7/tape.154 & apr 0 & 0 & & $\mathrm{~m}$ \\
\hline $\operatorname{cs} 135$ & 5531 & 1 & 0 & 55135 & aug98 & wright, s & ornl, hedl & rev7/tape.154 & apr 0 & $0 \mid$ & & $\mathrm{m}$ \\
\hline $\operatorname{cs} 136$ & 5534 & 0 & 0 & 55136 & apr74 & schenter & hedl & t105 & feb90 & 0 & $\mathrm{~s}$ & \\
\hline $\operatorname{cs} 137$ & 5537 & 0 & 0 & 55137 & apr74 & schenter & hedl & t105 & feb90 & 01 & & \\
\hline cu63 & 2925 & 4 & 0 & 29063 & feb98 & a.koning, & 3 lanl, orn & rev6/tape. 150 & aug99 & r3 & & \\
\hline cu65 & 2931 & 4 & 0 & 29065 & feb98 & a.koning, & 5 lanl, orn & rev6/tape. 150 & aug99 & r3 & & \\
\hline$d(d 20)$ & 11 & 0 & 0 & 111 & $\operatorname{dec} 69$ & koppel, ho & ga & t118 & may90 & 0 & & \\
\hline dy160 & 6637 & 1 & 0 & 66160 & oct74 & wright, s & ornl, hed & rev7/tape. 154 & apr 0 & 01 & & $\mathrm{~m}$ \\
\hline dy161 & 6640 & 1 & 0 & 66161 & oct74 & wright, s & ornl, hed & rev7/tape.154 & apr 0 & $0 \mid$ & & $\mathrm{m}$ \\
\hline dy162 & 6643 & 1 & 0 & 66162 & oct74 & wright, s & ornl, hed & rev7/tape.154 & apr 0 & 01 & & $\mathrm{~m}$ \\
\hline dy163 & 6646 & 1 & 0 & 66163 & oct74 & wright, s & ornl, hed & rev7/tape.154 & apr 0 & $0 \mid$ & & $\mathrm{m}$ \\
\hline dy164 & 6649 & 1 & 0 & 66164 & jun67 & wright, 1 & ornl, bnw & rev7/tape.154 & apr 0 & $0 \mid$ & & $\mathrm{m}$ \\
\hline er166 & 6837 & 1 & 0 & 68166 & $\operatorname{dec} 88$ & r. q. wri & ornl, hed & t103 & jan90 & 0 & & $\mathrm{~m}$ \\
\hline er167 & 6840 & 1 & 0 & 68167 & $\operatorname{dec} 88$ & r.q. wrig & ornl, hed & t103 & jan90 & 0 & & $\mathrm{~m}$ \\
\hline es253 & 9913 & 0 & 0 & 99253 & jul76 & kinsey, be & bnl,srl & t110 & feb90 & $0 \mid$ & $\mathrm{s}$ & \\
\hline eu151 & 6325 & 1 & 0 & 63151 & apr86 & p.g.young & $\operatorname{lanl}$ & t103 & jan90 & 0 & & $\mathrm{~m}$ \\
\hline eu152 & 6328 & 1 & 0 & 63152 & $\operatorname{dec} 88$ & r. q. wri & ornl, bnl & t103 & jan90 & 01 & & $\mathrm{~m}$ \\
\hline eu153 & 6331 & 2 & 0 & 63153 & aug99 & s.y.oh an & bnl+kaeri & rev7/tape. 154 & apr 0 & $0 \mid$ & & $\mathrm{m}$ \\
\hline eu154 & 6334 & 2 & 0 & 63154 & may89 & r.q.wrigh & ornl,bnl & rev7/tape. 154 & apr 0 & r1 & & $\mathrm{m}$ \\
\hline eu155 & 6337 & 3 & 0 & 63155 & $\operatorname{dec} 88$ & wright, pr & ornl, hed & rev7/tape.154 & apr 0 & 0 & & $\mathrm{~m}$ \\
\hline eu156 & 6340 & 0 & 0 & 63156 & apr74 & schenter & hedl & t106 & feb90 & 01 & & \\
\hline eu157 & 6343 & 0 & 0 & 63157 & apr74 & r.e.schen & hedl & t106 & feb90 & $0 \mid$ & & \\
\hline f19 & 925 & 1 & 0 & 9019 & jun90 & z.x.zhao, & cndc, ornl & t115 & jun90 & 0 & & \\
\hline fe54 & 2625 & 5 & 0 & 26054 & sep96 & m.b.chadw & 4 lanl, orn & rev6/tape. 148 & aug99 & r3 & & \\
\hline fe56 & 2631 & 3 & 0 & 26056 & sep96 & m.b.chadw & 6 lanl, orn & rev6/tape. 148 & aug99 & r2 & & \\
\hline fe57 & 2634 & 3 & 0 & 26057 & sep96 & m.b.chadw & 7 lanl, orn & rev6/tape. 148 & aug99 & r2 & & \\
\hline fe58 & 2637 & 3 & 0 & 26058 & nov89 & hetrick, $\mathrm{f}$ & ornl & rev5/tape. 140 & sep98 & $r 2$ & & \\
\hline ga & 3100 & 0 & 0 & 31000 & may80 & howerton, & llnl, lanl & t102 & jan90 & $0 \mid$ & & \\
\hline gd152 & 6425 & 1 & 0 & 64152 & $\operatorname{dec} 94$ & r.q.wrigh & ornl, jndc & rev4/tape.137 & & 0 & & $\mathrm{~m}$ \\
\hline $\operatorname{gd154}$ & 6431 & 1 & 0 & 64154 & $\operatorname{dec} 74$ & r.q.wrigh & ornl, jndc & rev4/tape.137 & & $0 \mid$ & & $\mathrm{m}$ \\
\hline gd155 & 6434 & 0 & 0 & 64155 & jan77 & b.a.magur & bnl & t106 & feb90 0 & $0 \mid$ & & $\mathrm{m}$ \\
\hline gd156 & 6437 & 0 & 0 & 64156 & jan77 & b.a.magur & $\mathrm{bnl}$ & t106 & feb90 & 0 & & $\mathrm{~m}$ \\
\hline gd157 & 6440 & 0 & 0 & 64157 & jan77 & b.a.magur & bnl & t106 & feb90 & 0 & & $\mathrm{~m}$ \\
\hline gd158 & 6443 & 0 & 0 & 64158 & jan77 & b.a.magur & $\mathrm{bnl}$ & t106 & feb90 & $0 \mid$ & & $\mathrm{m}$ \\
\hline gd160 & 6449 & 0 & 0 & 64160 & jan77 & b.a.magur & bnl & t106 & feb90 & 0 & & $\mathrm{~m}$ \\
\hline ge72 & 3231 & 0 & 0 & 32072 & apr74 & r.e.schen & hedl & t102 & jan90 & 0 & $\mathrm{~s}$ & \\
\hline ge73 & 3234 & 2 & 0 & 32073 & apr74 & r.e.schen & hedl & rev2/tape. 129 & jun93 & $r 1$ & $\mathrm{~s}$ & \\
\hline ge74 & 3237 & 0 & 0 & 32074 & apr74 & r.e.schen & hedl & $\mathrm{t} 102$ & jan90 & 0 & $\mathrm{~s}$ & \\
\hline ge76 & 3243 & 0 & 0 & 32076 & apr 74 & r.e.schen & hedl & $\mathrm{t} 102$ & jan90 & 01 & $\mathrm{~s}$ & \\
\hline graphite & 31 & 1 & 0 & 131 & apr93 & macfarlan & $\operatorname{lanl}$ & rev3/tape.133 & apr95 & 01 & & \\
\hline h (ch2) & 37 & 0 & 0 & 137 & $\operatorname{dec} 69$ & koppel, ho & ga & t118 & may 90 & 0 & & \\
\hline$h(h 20)$ & 1 & 1 & 0 & 101 & apr93 & macfarlan & $\operatorname{lanl}$ & rev3/tape. 132 & apr95 & 01 & & \\
\hline$h(z r h)$ & 7 & 1 & 0 & 107 & apr93 & macfarlan & $\operatorname{lanl}$ & rev3/tape.132 & apr95 & 0 & & \\
\hline h1 & 125 & 4 & 0 & 1001 & feb98 & g.m.hale, & $\operatorname{lanl}$ & rev6/tape.145 & aug99 & r3i & & \\
\hline h2 & 128 & 4 & 0 & 1002 & feb97 & p.g.young & $\operatorname{lanl}$ & rev6/tape. 145 & aug99 & r3 & & \\
\hline he3 & 225 & 2 & 0 & 2003 & may90 & g.hale, d & $\operatorname{lanl}$ & $\mathrm{t} 120$ & sep91 & $r 1$ & & \\
\hline he4 & 228 & 0 & 0 & 2004 & oct73 & nisley, h & $\operatorname{lanl}$ & t101 & jan90 & 0 & & \\
\hline hf & 7200 & 0 & 0 & 72000 & apr76 & m.drake, d & sai & t106 & feb90 & 0 & $\mathrm{~s}$ & \\
\hline hf174 & 7225 & 2 & 0 & 72174 & apr76 & r.q.wrigh & ornl, sai, & rev2/tape. 127 & jun93 & $r 1$ & & $\mathrm{~m}$ \\
\hline hf176 & 7231 & 2 & 0 & 72176 & apr76 & r.q.wrigh & ornl,sai, & rev2/tape. 127 & jun93 & r1 & & $\mathrm{m}$ \\
\hline hf177 & 7234 & 2 & 0 & 72177 & apr76 & r.q.wrigh & ornl,sai, & rev2/tape. 127 & jun93 & $r 1 \mid$ & & $\mathrm{m}$ \\
\hline hf178 & 7237 & 2 & 0 & 72178 & aug76 & r.q.wrigh & ornl,sai, & rev2/tape. 127 & jun93 & $r 1 \mid$ & & $\mathrm{m}$ \\
\hline hf179 & 7240 & 2 & 0 & 72179 & apr76 & r.q.wrigh & ornl,sai, & rev2/tape. 127 & jun93 & $r 1 \mid$ & & $\mathrm{m}$ \\
\hline hf180 & 7243 & 2 & 0 & 72180 & apr76 & r.q.wrigh & ornl,sai, & rev2/tape. 127 & jun93 & r1 & & $\mathrm{m}$ \\
\hline ho165 & 6725 & 2 & 0 & 67165 & apr88 & p.g.young & $\operatorname{lanl}$ & rev5/tape. 143 & sep98 & $r 1$ & $\mathrm{~s}$ & \\
\hline
\end{tabular}




\begin{tabular}{|c|c|c|c|c|c|c|c|c|c|c|c|c|}
\hline symbol & $\begin{array}{c}\text { mat } \\
----\end{array}$ & mod & $\begin{array}{l}\text { rel } \\
---\end{array}$ & $\begin{array}{r}z a \\
----\end{array}$ & $\begin{array}{l}\text { evdt } \\
-----\end{array}$ & evaluators & $\begin{array}{r}\text { org } \\
------\end{array}$ & $\begin{array}{r}\text { source } \\
----1\end{array}$ & dist & $\begin{array}{l}r v \mid: \\
--1\end{array}$ & $\begin{array}{ll}1 \mathrm{bw} \\
---\end{array}$ & $\begin{array}{l}\text { mlbw } \\
----\end{array}$ \\
\hline i127 & 5325 & 1 & 0 & 53127 & $\operatorname{mar} 91$ & p.g.young & $\operatorname{lan} 1$ & rev2/tape. 127 & jun93 & $0 \mid$ & & $\mathrm{m}$ \\
\hline i129 & 5331 & 0 & 0 & 53129 & feb80 & schenter & hedl, rcn & t105 & feb90 & $0 \mid$ & $\mathrm{s}$ & \\
\hline i130 & 5334 & 0 & 0 & 53130 & apr74 & schenter & hedl & t105 & feb90 & 0 & & \\
\hline i131 & 5337 & 0 & 0 & 53131 & apr74 & schenter & hedl & t105 & feb90 & 0 & & \\
\hline i135 & 5349 & 0 & 0 & 53135 & apr74 & schenter & hedl & t105 & feb90 & 0 & & \\
\hline in & 4900 & 1 & 0 & 49000 & feb90 & a.smith, d & anl & t116 & jun90 & $0 \mid$ & $\mathrm{s}$ & \\
\hline in113 & 4925 & 0 & 0 & 49113 & apr74 & r.e.schen & hedl & t116 & jun90 & 0 & $\mathrm{~s}$ & \\
\hline in115 & 4931 & 1 & 0 & 49115 & $\operatorname{mar} 90$ & f.schmitt & hedl, anl & t116 & jun90 & 0 & $\mathrm{~s}$ & \\
\hline ir191 & 7725 & 1 & 0 & 77191 & $\operatorname{mar} 95$ & r.q.wrigh & ornl & rev4/tape.137 & & 0 & & $\mathrm{~m}$ \\
\hline $\operatorname{ir193}$ & 7731 & 1 & 0 & 77193 & $\operatorname{mar} 95$ & r.q.wrigh & ornl & rev4/tape.137 & & 0 & & $\mathrm{~m}$ \\
\hline $\mathrm{k}$ & 1900 & 0 & 0 & 19000 & feb67 & m.k.drake & gga & t101 & jan90 & 0 & & \\
\hline k41 & 1931 & 0 & 0 & 19041 & jan79 & $\operatorname{mann}$ & hedl & t101 & jan90 & 01 & & \\
\hline $\mathrm{kr} 78$ & 3625 & 0 & 0 & 36078 & apr78 & a.prince & bnl & t102 & jan90 & 0 & s & \\
\hline $\operatorname{kr} 80$ & 3631 & 0 & 0 & 36080 & apr78 & a.prince & $\mathrm{bnl}$ & t102 & jan90 & $0 \mid$ & $\mathrm{s}$ & \\
\hline $\operatorname{kr} 82$ & 3637 & 0 & 0 & 36082 & apr78 & a.prince & bnl & t102 & jan90 & 0 & $\mathrm{~s}$ & \\
\hline kr83 & 3640 & 0 & 0 & 36083 & apr78 & a.prince & bnl & t102 & jan90 & 0 & $\mathrm{~s}$ & \\
\hline kr84 & 3643 & 0 & 0 & 36084 & apr78 & a.prince & bnl & t102 & jan90 & $0 \mid$ & $\mathrm{s}$ & \\
\hline kr85 & 3646 & 0 & 0 & 36085 & apr74 & schenter & hedl & t102 & jan90 & 01 & & \\
\hline kr86 & 3649 & 0 & 0 & 36086 & jul72 & a.prince & bnl & t102 & jan90 & 0 & $\mathrm{~s}$ & \\
\hline la139 & 5728 & 2 & 0 & 57139 & feb80 & r.e.schen & hedl, $r \mathrm{cn}$ & t120 & sep91 & r1 & $\mathrm{s}$ & \\
\hline la140 & 5731 & 0 & 0 & 57140 & apr74 & schenter & hedl & t105 & feb90 & 0 & & \\
\hline lch4 & 33 & 1 & 0 & 133 & apr93 & macfarlan & $\operatorname{lanl}$ & rev3/tape.133 & apr95 & $0 \mid$ & & \\
\hline li6 & 325 & 2 & 0 & 3006 & apr89 & g.m.hale, & $\operatorname{lanl}$ & t120 & sep91 & $r 1$ & & \\
\hline $1 i 7$ & 328 & 1 & 0 & 3007 & aug 88 & p.g.young & $\operatorname{lanl}$ & t100 & jan90 & 01 & & \\
\hline lu175 & 7125 & 1 & 0 & 71175 & $\operatorname{mar9} 98$ & r.q. wrig & ornl, bnw & rev7/tape. 154 & apr 0 & $r 1$ & & $\mathrm{~m}$ \\
\hline lu176 & 7128 & 1 & 0 & 71176 & $\operatorname{mar} 98$ & r.q. wrig & ornl, bnw & rev7/tape.154 & apr 0 & $r 1 \mid$ & & $\mathrm{m}$ \\
\hline $\mathrm{mg}$ & 1200 & 0 & 0 & 12000 & feb78 & d.c.larso & ornl & t101 & jan90 & 0 & & \\
\hline mg24 & 1225 & 0 & 0 & 12024 & nov79 & mann, lars & hedl, ornl & t101 & jan90 & 0 & & \\
\hline $\operatorname{mn} 55$ & 2525 & 2 & 0 & 25055 & $\operatorname{mar} 88$ & k. shibata & jaeri, orn & rev5/tape. 140 & sep98 & r1 & & $\mathrm{m}$ \\
\hline mo & 4200 & 0 & 0 & 42000 & feb79 & howerton, & llnl, hedl & t104 & jan90 & 0 & $\mathrm{~s}$ & \\
\hline mo100 & 4249 & 0 & 0 & 42100 & feb80 & schenter, & hedl,rcn & t104 & jan90 & 01 & $\mathrm{~s}$ & \\
\hline mo92 & 4225 & 0 & 0 & 42092 & feb80 & schenter, & hedl, ren & t104 & jan90 & 0 & $\mathrm{~s}$ & \\
\hline mo94 & 4231 & 0 & 0 & 42094 & feb80 & r.e.schen & hedl, rcn & t104 & jan90 & 0 & $\mathrm{~s}$ & \\
\hline mo95 & 4234 & 0 & 0 & 42095 & feb80 & r.e.schen & hedl, rcn & t104 & jan90 & 01 & $\mathrm{~s}$ & \\
\hline $\operatorname{mo9} 6$ & 4237 & 0 & 0 & 42096 & feb80 & r.e.schen & hedl, rcn & t104 & jan90 & $0 \mid$ & $\mathrm{s}$ & \\
\hline mo97 & 4240 & 0 & 0 & 42097 & feb80 & r.e.schen & hedl, rcn & t104 & jan90 & 0 & $\mathrm{~s}$ & \\
\hline mo98 & 4243 & 0 & 0 & 42098 & feb80 & schenter, & hedl, rcn & t104 & jan90 & $0 \mid$ & $\mathrm{s}$ & \\
\hline mo99 & 4246 & 0 & 0 & 42099 & apr74 & schenter & hedl & t104 & jan90 & 0 & & \\
\hline n14 & 725 & 4 & 0 & 7014 & jun97 & m.b.chadw & 4 lanl & rev6/tape.145 & aug99 & $r 3 \mid$ & & \\
\hline n15 & 728 & 1 & 0 & 7015 & sep83 & e.arthur, & lanl & t116 & jun90 & 0 & & \\
\hline na23 & 1125 & 2 & 0 & 11023 & $\operatorname{dec} 77$ & d. c. lar & ornl & t 120 & sep91 & $r 1 \mid$ & & $\mathrm{m}$ \\
\hline nb93 & 4125 & 3 & 0 & 41093 & $\operatorname{dec} 97$ & m.chadwic & 3 lanl, anl & rev6/tape. 150 & aug99 & $\mathrm{r} 2$ & $\mathrm{~s}$ & \\
\hline nb94 & 4128 & 0 & 0 & 41094 & apr74 & schenter & hedl & t104 & jan90 & 0 & $\mathrm{~s}$ & \\
\hline nb95 & 4131 & 0 & 0 & 41095 & apr74 & schenter & hedl & t104 & jan90 & 01 & & \\
\hline nd142 & 6025 & 0 & 0 & 60142 & apr74 & r.e.schen & hedl & t105 & feb90 & 0 & $\mathrm{~s}$ & \\
\hline nd143 & 6028 & 2 & 0 & 60143 & oct74 & r.q.wrigh & ornl, hedl & rev2/tape. 127 & jun93 & $r 1$ & & $\mathrm{~m}$ \\
\hline nd144 & 6031 & 0 & 0 & 60144 & feb80 & schenter & hedl & t105 & feb90 & 0 & $\mathrm{~s}$ & \\
\hline nd145 & 6034 & 2 & 0 & 60145 & feb80 & r.q.wrigh & ornl, hedl & rev2/tape. 127 & jun93 & $r 1 \mid$ & & $\mathrm{m}$ \\
\hline nd146 & 6037 & 0 & 0 & 60146 & feb80 & schenter, & hedl, bnl+ & t105 & feb90 & 0 & $\mathrm{~s}$ & \\
\hline nd147 & 6040 & 2 & 0 & 60147 & $\operatorname{dec} 88$ & wright, s & ornl, hed & t120 & sep91 & r1 & $\mathrm{s}$ & \\
\hline nd148 & 6043 & 0 & 0 & 60148 & feb80 & schenter, & hedl, bnl+ & t105 & feb90 & 0 & $\mathrm{~s}$ & \\
\hline nd150 & 6049 & 0 & 0 & 60150 & feb80 & schenter, & hedl, bnl+ & t105 & feb90 & 0 & $\mathrm{~s}$ & \\
\hline ni58 & 2825 & 3 & 0 & 28058 & sep97 & s.chiba, m & 8 lanl, orn & rev6/tape.149 & aug99 & r2 & & \\
\hline ni59 & 2828 & 1 & 0 & 28059 & jan83 & f.m.mann & hedl & t113 & apr90 & 0 & & \\
\hline ni60 & 2831 & 3 & 0 & 28060 & sep97 & s.chiba, m & 0 lanl, orn & rev6/tape.149 & aug99 & r2 & & \\
\hline ni61 & 2834 & 4 & 0 & 28061 & sep97 & s.chiba, m & 1 lanl, orn & rev6/tape. 149 & aug99 & r3 & & \\
\hline ni62 & 2837 & 4 & 0 & 28062 & sep97 & s.chiba, m & 2 lanl, orn & rev6/tape.149 & aug99 & r3 & & \\
\hline ni64 & 2843 & 3 & 0 & 28064 & sep97 & s.chiba, m & 4 lanl, orn & rev6/tape.149 & aug99 & r2 & & \\
\hline np237 & 9346 & 2 & 0 & 93237 & apr90 & p.young, & $\operatorname{lan} 1$ & t121 & sep91 & r1 & $\mathrm{s}$ & \\
\hline np238 & 9349 & 2 & 0 & 93238 & aug75 & benjamin & srl & rev2/tape.129 & jun93 & $r 1 \mid$ & $\mathrm{s}$ & \\
\hline np239 & 9352 & 1 & 0 & 93239 & $\operatorname{dec} 88$ & r. q. wri & ornl & t108 & feb90 & 0 & & \\
\hline o16 & 825 & 2 & 0 & 8016 & jun96 & m.b.chadw & $6 \operatorname{lan} 1$ & rev6/tape.145 & aug99 & $r 1$ & & \\
\hline ०17 & 828 & 0 & 0 & 8017 & jan78 & b.a.magur & bnl & t101 & jan90 & 0 & & \\
\hline orthod & 13 & 1 & 0 & 113 & apr93 & macfarlan & lanl & rev3/tape.132 & apr95 & $0 \mid$ & & \\
\hline orthoh & 3 & 1 & 0 & 103 & apr93 & macfarlan & $\operatorname{lan} 1$ & rev3/tape.132 & apr95 & 0 & & \\
\hline p31 & 1525 & 1 & 0 & 15031 & $\operatorname{dec} 97$ & m.chadwic & 1 lanl, lln & rev6/tape.146 & aug99 & $r 1 \mid$ & & \\
\hline pa231 & 9131 & 1 & 0 & 91231 & nov77 & mann & hedl & t109 & feb90 & 01 & $\mathrm{~s}$ & \\
\hline pa233 & 9137 & 0 & 0 & 91233 & may78 & mathews, $\mathrm{k}$ & $g a, b n l, l a$ & t110 & feb90 & 01 & $\mathrm{~s}$ & \\
\hline parad & 12 & 1 & 0 & 112 & apr93 & macfarlan & $\operatorname{lan} 1$ & rev3/tape. 132 & apr95 & 01 & & \\
\hline parah & 2 & 1 & 0 & 102 & apr93 & macfarlan & $\operatorname{lanl}$ & rev3/tape.132 & apr95 & 0 & & \\
\hline pb206 & 8231 & 2 & 0 & 82206 & aug96 & m.b.chadw & 6 lanl, orn & rev6/tape.152 & aug99 & $r 1 \mid$ & & \\
\hline $\mathrm{pb} 207$ & 8234 & 3 & 0 & 82207 & aug96 & m.b.chadw & 7 lanl, orn & rev6/tape. 152 & aug99 & r2 & & \\
\hline pb208 & 8237 & 3 & 0 & 82208 & aug96 & m.b.chadw & 8 lanl, orn & rev6/tape. 152 & aug99 & r2 & & \\
\hline pd102 & 4625 & 1 & 0 & 46102 & jan96 & p. g. you & $\operatorname{lanl}$ & rev5/tape.141 & sep98 & 0 & & \\
\hline pd104 & 4631 & 1 & 0 & 46104 & jan96 & p. g. you & $\operatorname{lanl}$ & rev5/tape.141 & sep98 & 0 & & $\mathrm{~m}$ \\
\hline pd105 & 4634 & 2 & 0 & 46105 & jan96 & p. g. you & $\operatorname{lanl}$ & rev5/tape.141 & sep98 & 0 & & $\mathrm{~m}$ \\
\hline pd106 & 4637 & 1 & 0 & 46106 & jan96 & p. g. you & $\operatorname{lanl}$ & rev5/tape.141 & sep98 & $0 \mid$ & & $\mathrm{m}$ \\
\hline pd107 & 4640 & 1 & 0 & 46107 & $\operatorname{dec} 89$ & r.q.wrigh & ornl, hedl & $\mathrm{t} 103$ & jan90 & 0 & & $\mathrm{~m}$ \\
\hline pd108 & 4643 & 1 & 0 & 46108 & jan96 & p. g. you & $\operatorname{lanl}$ & rev5/tape.141 & sep98 & 0 & & $\mathrm{~m}$ \\
\hline pd110 & 4649 & 1 & 0 & 46110 & jan96 & p. g. you & $\operatorname{lanl}$ & rev5/tape.141 & sep98 & 0 & & \\
\hline pm147 & 6149 & 2 & 0 & 61147 & apr89 & r.q.wrigh & ornl, hedl & $\mathrm{t} 120$ & sep91 & $r 1$ & & $\mathrm{~m}$ \\
\hline pm148 & 6152 & 0 & 0 & 61148 & apr74 & schenter & hedl & t106 & feb90 & 0 & & \\
\hline pm148m & 6153 & 0 & 0 & 61148 & apr74 & schenter & mhedl & t106 & feb90 & $0 \mid$ & $\mathrm{s}$ & \\
\hline pm149 & 6155 & 0 & 0 & 61149 & apr 74 & schenter & hedl & t106 & feb9o & 0 & & \\
\hline pm151 & 6161 & 0 & 0 & 61151 & apr74 & schenter & hedl & t106 & feb90 & 01 & & \\
\hline
\end{tabular}




\begin{tabular}{|c|c|c|c|c|c|c|c|c|c|c|c|c|}
\hline symbol & $\begin{array}{r}\text { mat } \\
----\end{array}$ & $\begin{array}{l}\bmod \\
---\end{array}$ & $\begin{array}{l}\text { rel } \\
---\end{array}$ & $\begin{array}{r}z a \\
----\end{array}$ & $\begin{array}{r}\text { evdt } \\
----\end{array}$ & evaluators & $\begin{array}{r}\text { org } \\
------\end{array}$ & $\begin{array}{r}\text { source } \\
-\end{array}$ & dist & $\begin{array}{l}r v \mid: \\
--1\end{array}$ & ibw & $\begin{array}{l}\text { mlbw } \\
----\end{array}$ \\
\hline pr141 & 5925 & 1 & 0 & 59141 & aug99 & s.y.oh, s. & bnl, kaeri & rev7/tape. 154 & apr 0 & $0 \mid$ & & $\mathrm{m}$ \\
\hline pr142 & 5928 & 0 & 0 & 59142 & apr74 & schenter & hedl & t105 & feb90 & $0 \mid$ & & \\
\hline pr143 & 5931 & 0 & 0 & 59143 & apr74 & schenter & hedl & t105 & feb90 & 0 & & \\
\hline pu236 & 9428 & 1 & 0 & 94236 & sep95 & r. q. wri & ornl,mapi & rev4/tape.137 & & 0 & & $\mathrm{~m}$ \\
\hline pu237 & 9431 & 0 & 0 & 94237 & apr78 & $\operatorname{mann}$ and & hedl & t110 & feb90 & $0 \mid$ & & \\
\hline pu238 & 9434 & 1 & 0 & 94238 & apr78 & mann, sche & hedl,ai, + & t109 & feb90 & 0 & $\mathrm{~s}$ & \\
\hline pu239 & 9437 & 3 & 0 & 94239 & apr89 & p.young, & $\operatorname{lan} 1$ & rev5/tape. 142 & sep98 & r2 & & \\
\hline pu240 & 9440 & 3 & 0 & 94240 & aug 86 & 1.w. west & ornl & rev2/tape. 128 & jun93 & r2 & & $\mathrm{m}$ \\
\hline pu241 & 9443 & 3 & 0 & 94241 & oct 88 & 1.weston, & ornl & rev3/tape.135 & sep91 & r2 & & \\
\hline pu242 & 9446 & 1 & 0 & 94242 & oct78 & mann, benj & hedl,srl, & t109 & feb90 & 0 & $\mathrm{~s}$ & \\
\hline pu243 & 9449 & 2 & 0 & 94243 & jul76 & kinsey-as & bnl, srl, + & rev2/tape.129 & jun93 & r1| & $\mathrm{s}$ & \\
\hline pu244 & 9452 & 0 & 0 & 94244 & apr78 & mann, sche & hedl,srl & t110 & feb90 & $0 \mid$ & $\mathrm{s}$ & \\
\hline rb85 & 3725 & 0 & 0 & 37085 & oct79 & a. prince & bnl-brc & t102 & jan90 & 0 & & $\mathrm{~m}$ \\
\hline rb86 & 3728 & 0 & 0 & 37086 & apr74 & schenter & hedl & t102 & jan90 & $0 \mid$ & & \\
\hline rb87 & 3731 & 0 & 0 & 37087 & oct79 & a. prince & $\mathrm{bnl}-\mathrm{brc}$ & t102 & jan90 & 0 & & $\mathrm{~m}$ \\
\hline re185 & 7525 & 1 & 0 & 75185 & $\operatorname{mar} 90$ & 1.w.westo & ornl, lanl & t115 & jun90 & 0 & & $\mathrm{~m}$ \\
\hline re187 & 7531 & 1 & 0 & 75187 & $\operatorname{mar} 90$ & 1.w.westo & ornl, lanl & t115 & jun90 & $0 \mid$ & & $\mathrm{m}$ \\
\hline rh103 & 4525 & 0 & 0 & 45103 & nov78 & schenter, & hedl, baw & t104 & jan90 & 01 & $\mathrm{~s}$ & \\
\hline rh105 & 4531 & 0 & 0 & 45105 & apr74 & schenter & hedl & t104 & jan90 & 0 & & \\
\hline ru100 & 4437 & 0 & 0 & 44100 & feb80 & r.e.schen & hedl, ren & t104 & jan90 & 01 & $\mathrm{~s}$ & \\
\hline ru101 & 4440 & 2 & 0 & 44101 & feb80 & r.q.wrigh & ornl, hedl & rev2/tape.127 & jun93 & $r 1$ & & $\mathrm{~m}$ \\
\hline ru102 & 4443 & 2 & 0 & 44102 & feb80 & r.q.wrigh & ornl, hedl & rev2/tape.127 & jun93 & $r 1$ & & $\mathrm{~m}$ \\
\hline ru103 & 4446 & 0 & 0 & 44103 & apr74 & schenter & hedl & t104 & jan90 & 0 & & \\
\hline ru104 & 4449 & 0 & 0 & 44104 & feb80 & r.e.schen & hedl, $r \mathrm{cn}$ & t104 & jan90 & 01 & $\mathrm{~s}$ & \\
\hline ru105 & 4452 & 0 & 0 & 44105 & apr74 & schenter & hedl & t104 & jan90 & 0 & & \\
\hline ru106 & 4455 & 0 & 0 & 44106 & apr74 & schenter & hedl & t104 & jan90 & 0 & & \\
\hline ru96 & 4425 & 0 & 0 & 44096 & feb80 & f.m.mann & hedl & t104 & jan90 & 01 & & \\
\hline ru98 & 4431 & 0 & 0 & 44098 & feb80 & f.m.mann & hedl & t104 & jan90 & 0 & & \\
\hline ru99 & 4434 & 0 & 0 & 44099 & $\operatorname{apr} 74$ & r.e.schen & hedl & t104 & jan90 & 0 & $\mathrm{~s}$ & \\
\hline $\mathrm{s}$ & 1600 & 0 & 0 & 16000 & apr79 & divadeena & bnl & t101 & jan90 & 01 & & $\mathrm{~m}$ \\
\hline s32 & 1625 & 0 & 0 & 16032 & oct 77 & howerton & $\ln 1$ & t101 & jan90 & 0 & & \\
\hline sb121 & 5125 & 0 & 0 & 51121 & feb80 & r.e.schen & hedl, rcn & t105 & feb90 & $0 \mid$ & $\mathrm{s}$ & \\
\hline sb123 & 5131 & 0 & 0 & 51123 & feb80 & r.e.schen & hedl, ren & t105 & feb90 & 0 & $\mathrm{~s}$ & \\
\hline sb124 & 5134 & 0 & 0 & 51124 & apr74 & schenter & hedl & t105 & feb90 & 0 & & \\
\hline sb125 & 5137 & 0 & 0 & 51125 & apr74 & schenter & hedl & t105 & feb90 & 01 & & \\
\hline sb126 & 5140 & 0 & 0 & 51126 & apr74 & schenter & hedl & t105 & feb90 & $0 \mid$ & & \\
\hline $\operatorname{sc} 45$ & 2125 & 1 & 0 & 21045 & jul92 & a.b.smith & anl, llnl & rev2/tape.127 & jun93 & 0 & & $\mathrm{~m}$ \\
\hline sch4 & 34 & 1 & 0 & 134 & apr93 & macfarlan & $\operatorname{lanl}$ & rev3/tape.133 & apr95 & 0 & & \\
\hline se74 & 3425 & 0 & 0 & 34074 & feb80 & f.m.mann & hedl & t102 & jan90 & 0 & $\mathrm{~s}$ & \\
\hline se76 & 3431 & 0 & 0 & 34076 & apr74 & r.e.schen & hedl & t102 & jan90 & 0 & $\mathrm{~s}$ & \\
\hline se77 & 3434 & 0 & 0 & 34077 & apr74 & r.e.schen & hedl & t102 & jan90 & 01 & $\mathrm{~s}$ & \\
\hline se78 & 3437 & 0 & 0 & 34078 & apr74 & r.e.schen & hedl & t102 & jan90 & 0 & $\mathrm{~s}$ & \\
\hline se 80 & 3443 & 0 & 0 & 34080 & apr74 & r.e.schen & hedl & t102 & jan90 & 0 & $\mathrm{~s}$ & \\
\hline se82 & 3449 & 0 & 0 & 34082 & apr74 & r.e.schen & hedl & t102 & jan90 & 0 & $\mathrm{~s}$ & \\
\hline si & 1400 & 0 & 0 & 14000 & feb74 & larson, pe & ornl & t116 & jun90 & 01 & & \\
\hline $\operatorname{si28}$ & 1425 & 3 & 0 & 14028 & may96 & d.hetrick & ornl & rev5/tape.139 & sep98 & 01 & & \\
\hline $\operatorname{si29}$ & 1428 & 2 & 0 & 14029 & jun97 & m.b.chadw & 9 lanl, orn & rev6/tape.146 & aug99 & $r 1 \mid$ & & \\
\hline si30 & 1431 & 2 & 0 & 14030 & jun97 & m.b.chadw & 0 lanl, orn & rev6/tape.146 & aug99 & $r 1 \mid$ & & \\
\hline $\operatorname{sm} 144$ & 6225 & 2 & 0 & 62144 & feb80 & r.q. wrig & ornl, hedl & rev3/tape.134 & apr95 & $r 1$ & & $\mathrm{~m}$ \\
\hline $\operatorname{sm} 147$ & 6234 & 1 & 0 & 62147 & apr89 & wright, sc & ornl, hedl & $\mathrm{t} 103$ & jan90 & 0 & & $\mathrm{~m}$ \\
\hline $\operatorname{sm} 148$ & 6237 & 0 & 0 & 62148 & apr74 & schenter & hedl & $\mathrm{t} 106$ & feb90 & $0 \mid$ & & \\
\hline $\operatorname{sm} 149$ & 6240 & 1 & 0 & 62149 & aug99 & j.h.chang & bnl+kaeri & rev7/tape.154 & apr 0 & 0 & & $\mathrm{~m}$ \\
\hline $\operatorname{sm} 150$ & 6243 & 2 & 0 & 62150 & apr74 & r.q.wrigh & ornl, hedl & rev2/tape.127 & jun93 & rol & & $\mathrm{m}$ \\
\hline $\operatorname{sm} 151$ & 6246 & 2 & 0 & 62151 & $\operatorname{mar} 89$ & wright, s & ornl, hedl & $\mathrm{t} 120$ & sep91 & $r 1 \mid$ & & $\mathrm{m}$ \\
\hline $\operatorname{sm} 152$ & 6249 & 2 & 0 & 62152 & feb80 & r.q.wrigh & ornl, hedl & rev2/tape. 127 & jun93 & $r 1 \mid$ & & $\mathrm{m}$ \\
\hline $\operatorname{sm} 153$ & 6252 & 0 & 0 & 62153 & apr74 & schenter & hedl & t106 & feb90 & 0 & & \\
\hline $\operatorname{sm} 154$ & 6255 & 0 & 0 & 62154 & apr74 & r.e.schen & hedl & t106 & feb90 & 0 & $\mathrm{~s}$ & \\
\hline $\operatorname{sn} 112$ & 5025 & 2 & 0 & 50112 & feb80 & f.m.mann & hedl & t 120 & sep91 & r1 & $\mathrm{s}$ & \\
\hline $\operatorname{sn} 114$ & 5031 & 2 & 0 & 50114 & feb80 & f.m.mann & hedl & t 120 & sep91 & $r 1$ & $\mathrm{~s}$ & \\
\hline $\operatorname{sn} 115$ & 5034 & 0 & 0 & 50115 & apr74 & r.e.schen & hedl & t105 & feb90 & 0 & $\mathrm{~s}$ & \\
\hline $\operatorname{sn} 116$ & 5037 & 0 & 0 & 50116 & apr74 & r.e.schen & hedl & t105 & feb90 & 01 & $\mathrm{~s}$ & \\
\hline $\operatorname{sn} 117$ & 5040 & 0 & 0 & 50117 & apr74 & r.e.schen & hedl & t105 & feb90 & 0 & $\mathrm{~s}$ & \\
\hline $\operatorname{sn} 118$ & 5043 & 0 & 0 & 50118 & apr74 & r.e.schen & hedl & t105 & feb90 & 0 & $\mathrm{~s}$ & \\
\hline $\operatorname{sn} 119$ & 5046 & 0 & 0 & 50119 & apr74 & r.e.schen & hedl & t105 & feb90 & 0 & $\mathrm{~s}$ & \\
\hline $\operatorname{sn} 120$ & 5049 & 0 & 0 & 50120 & oct74 & r.e.schen & hedl & t105 & feb90 & 01 & $\mathrm{~s}$ & \\
\hline $\operatorname{sn} 122$ & 5055 & 0 & 0 & 50122 & oct74 & r.e.schen & hedl & t105 & feb90 & $0 \mid$ & $\mathrm{s}$ & \\
\hline $\operatorname{sn} 123$ & 5058 & 0 & 0 & 50123 & apr74 & schenter & hedl & t105 & feb90 & 01 & & \\
\hline $\operatorname{sn} 124$ & 5061 & 0 & 0 & 50124 & oct74 & r.e.schen & hedl & t105 & feb90 & 01 & $\mathrm{~s}$ & \\
\hline $\operatorname{sn} 125$ & 5064 & 0 & 0 & 50125 & apr74 & schenter & hedl & t105 & feb90 & 01 & & \\
\hline $\operatorname{sn} 126$ & 5067 & 0 & 0 & 50126 & apr74 & schenter & hedl & t105 & feb90 & 0 & & \\
\hline $\operatorname{sr} 84$ & 3825 & 0 & 0 & 38084 & feb80 & f.m.mann & hedl & t102 & jan90 & 01 & $\mathrm{~s}$ & \\
\hline $\operatorname{sr} 86$ & 3831 & 0 & 0 & 38086 & apr74 & r.e.schen & hedl & t102 & jan90 & 01 & $\mathrm{~s}$ & \\
\hline $\operatorname{sr} 87$ & 3834 & 0 & 0 & 38087 & apr74 & r.e.schen & hedl & t102 & jan90 & 01 & $\mathrm{~s}$ & \\
\hline $\operatorname{sr} 88$ & 3837 & 0 & 0 & 38088 & apr74 & r.e.schen & hedl & t102 & jan90 & 0 & $\mathrm{~s}$ & \\
\hline $\operatorname{sr} 89$ & 3840 & 0 & 0 & 38089 & apr74 & schenter & hedl & t102 & jan90 & 0 & & \\
\hline $\operatorname{sr} 90$ & 3843 & 0 & 0 & 38090 & apr74 & schenter & hedl & t102 & jan90 & 0 & & \\
\hline t3 & 131 & 0 & 0 & 1003 & feb65 & leona ste & $\operatorname{lanl}$ & t101 & jan90 & 0 & & \\
\hline ta181 & 7328 & 0 & 0 & 73181 & jan72 & howerton, & $\ln 1$ & t106 & feb9o & 0 & & $\mathrm{~m}$ \\
\hline ta182 & 7331 & 0 & 0 & 73182 & apr71 & j.otter, c & ai & t106 & feb90 & 01 & & $\mathrm{~m}$ \\
\hline 0159 & 6525 & 0 & 0 & 65159 & feb80 & r.e.schen & hedl, rcn & t106 & feb90 & 0 & $\mathrm{~s}$ & \\
\hline 0160 & 6528 & 0 & 0 & 65160 & apr74 & schenter & hedl & t106 & feb90 & 0 & & \\
\hline 999 & 4325 & 0 & 0 & 43099 & nov78 & schenter, & hedl, baw & t104 & jan90 & 0 & $\mathrm{~s}$ & \\
\hline 120 & 5225 & 0 & 0 & 52120 & feb80 & f.m.mann & hedl & t105 & feb90 & 0 & & \\
\hline 122 & 5231 & 0 & 0 & 52122 & apr74 & r.e.schen & hedl & t105 & feb9o & 0 & $\mathrm{~s}$ & \\
\hline 123 & 5234 & 0 & 0 & 52123 & apr74 & r.e.schen & hedl & t105 & feb90 & 01 & $\mathrm{~s}$ & \\
\hline
\end{tabular}




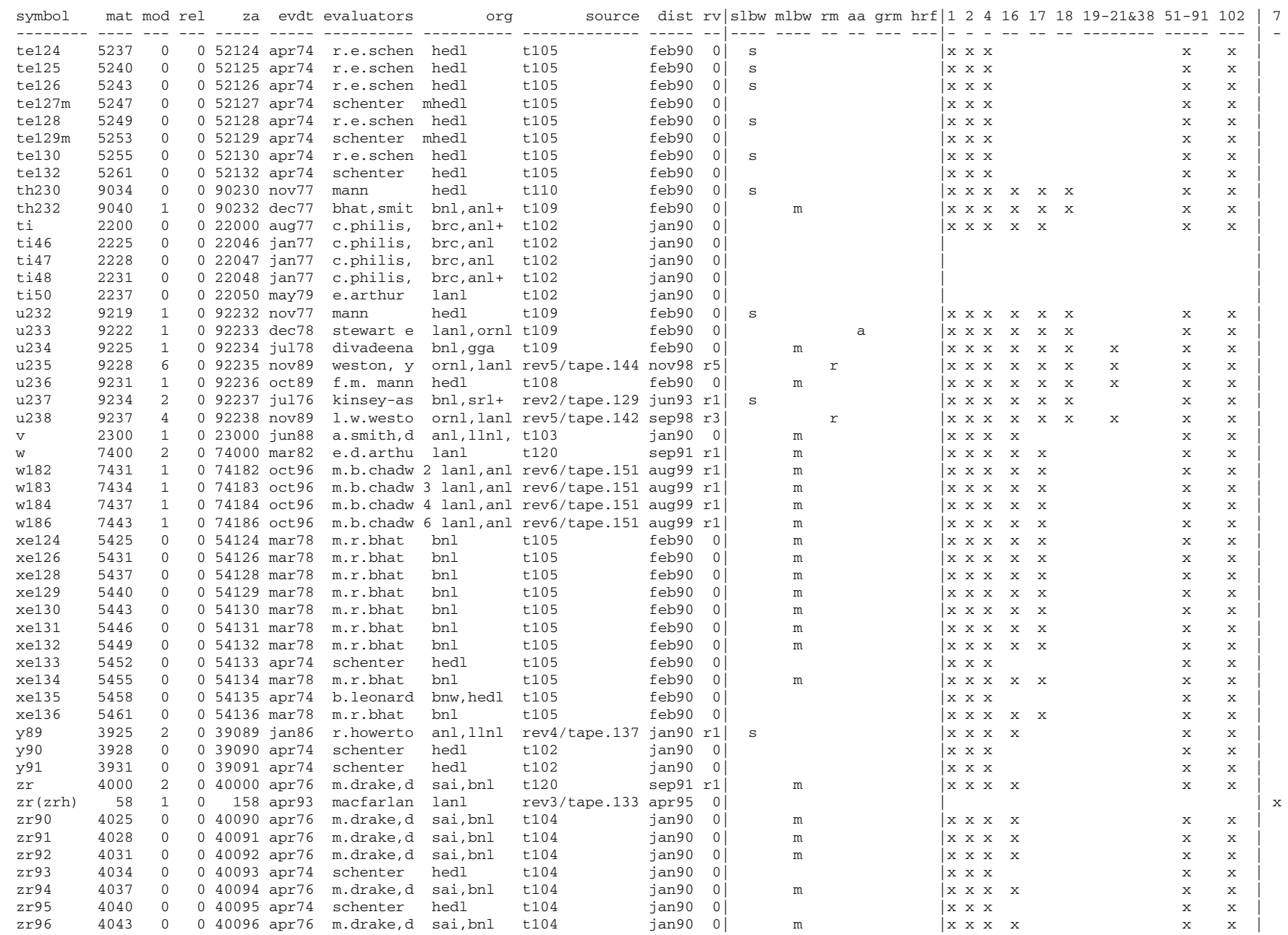




\section{APPENDIX B CONTENTS OF 238-GROUP ENDF/B-VI LIBRARY GENERATED WITH AMPX-2000}

The following is the directory of the 238-group ENDF/B-VI library that has been generated in support of the temperature-effects study. For each entry in the directory, the SCALE-identifier number is provided for each material followed by a descriptive title card. The first entry for each title card is the symbol associated with the material. Following the symbol information is the 4-digit ENDF material number and ZA number. The remaining information in the title card is a condensed description of the ENDF/B source information.

\begin{tabular}{|c|c|c|c|c|c|c|c|c|c|c|c|c|c|}
\hline Oentry & identifier & & & & & & $t i$ & t 1 & & & & & source \\
\hline 1 & 47107 & $\operatorname{ag} 107$ & 4725 & 47107 & ENDFB & V6 & REL0 & REV0 & MOD0 & AMPX & 06/08/01 & t104 & 25 \\
\hline 2 & 47109 & ag109 & 4731 & 47109 & ENDFB & V6 & RELO & REVO & MOD0 & AMPX & 06/08/01 & t104 & 25 \\
\hline 3 & 47111 & ag111 & 4737 & 47111 & ENDFB & V6 & RELO & REVO & MOD0 & AMPX & 06/08/01 & t104 & 25 \\
\hline 4 & 13027 & al27 & 1325 & 13027 & ENDFB & V6 & RELO & REV2 & MOD3 & AMPX & 06/08/01 & rev6/tape. 145 & 5 \\
\hline 5 & 95241 & $\operatorname{am} 241$ & 9543 & 95241 & ENDFB & V6 & RELO & REV2 & MOD3 & AMPX & 06/08/01 & rev3/tape.135 & 5 \\
\hline 6 & 95242 & am242 & 9546 & 95242 & ENDFB & V6 & RELO & REV1 & MOD2 & AMPX & 06/08/01 & t121 & \\
\hline 7 & 95601 & $\operatorname{am} 242 m$ & 9547 & 95601 & endfb & v6 & relo & rev1 & $\bmod 2$ & ampx & $08 / 06 / 01$ & t121 & \\
\hline 8 & 95243 & $\operatorname{am} 243$ & 9549 & 95243 & ENDFB & V6 & RELO & REV1 & MOD2 & AMPX & $06 / 08 / 01$ & rev5/tape.143 & \\
\hline 9 & 18040 & $\operatorname{ar} 40$ & 1837 & 18040 & ENDFB & V6 & RELO & REVO & MODO & AMPX] & INCOMPLETE & et101 & \\
\hline 10 & 33075 & as 75 & 3325 & 33075 & ENDFB & V6 & RELO & REVO & MOD0 & AMPX & 06/08/01 & t102 & \\
\hline 11 & 79197 & au197 & 7925 & 79197 & ENDFB & V6 & RELO & REV1 & MOD2 & AMPX & 06/08/01 & t120 & \\
\hline 12 & 5010 & b10 & 525 & 5010 & ENDFB & V6 & RELO & REV1 & MOD2 & AMPX & 06/08/01 & t120 & \\
\hline 13 & 5011 & b11 & 528 & 5011 & ENDFB & V6 & REL0 & REV0 & MOD1 & AMPX & $06 / 08 / 01$ & t100 & \\
\hline 14 & 56134 & ba134 & 5637 & 56134 & ENDFB & V6 & RELO & REVO & MOD2 & AMPX & 06/08/01 & rev7/tape.154 & \\
\hline 15 & 56135 & ba135 & 5640 & 56135 & ENDFB & V6 & RELO & REVO & MOD1 & AMPX & $06 / 08 / 01$ & t103 & \\
\hline 16 & 56136 & ba136 & 5643 & 56136 & ENDFB & V6 & RELO & REVO & MOD1 & AMPX & $06 / 08 / 01$ & t103 & \\
\hline 17 & 56137 & ba137 & 5646 & 56137 & ENDFB & V6 & RELO & REVO & MOD1 & AMPX & 06/08/01 & t103 & \\
\hline 18 & 56138 & ba138 & 5649 & 56138 & ENDFB & V6 & RELO & REV1 & MOD2 & AMPX & $06 / 08 / 01$ & rev3/tape.134 & 25 \\
\hline 19 & 56140 & ba140 & 5655 & 56140 & ENDFB & V6 & RELO & REVO & MOD0 & AMPX & $06 / 08 / 01$ & t105 & 25 \\
\hline 20 & 4009 & be9 & 425 & 4009 & ENDFB & V6 & RELO & REVO & MOD1 & AMPX & 06/08/01 & t100 & 25 \\
\hline 21 & 4309 & bemetal & 26 & 4309 & endfb & v6 & relo & revo & $\bmod 1$ & ampx & 08/06/01 & rev3/tape.133 & 25 \\
\hline 22 & 1601 & benzine & 40 & 1601 & endfb & v6 & relo & rev0 & $\bmod 0$ & ampx & 08/06/01 & t119 & 25 \\
\hline 23 & 6612 & C_benzine & 40 & 6612 & endfb & v6 & rel & rev & $\bmod$ & ampx & 08/06/01 & t119 & 25 \\
\hline 24 & 4509 & beo & 27 & 4509 & endfb & v6 & relo & rev0 & mod1 & ampx & 08/06/01 & rev3/tape.133 & 2 \\
\hline 25 & 8516 & o_beo & 27 & 8516 & endfb & v6 & rel & rev & $\bmod$ & ampx & 08/06/01 & rev3/tape.133 & 2 \\
\hline 26 & 83209 & bi209 & 8325 & 83209 & ENDFB & V6 & RELO & REVO & MOD2 & AMPX & 06/08/01 & rev7/tape.155 & 25 \\
\hline 27 & 97249 & bk249 & 9752 & 97249 & ENDFB & V6 & RELO & REVO & MOD1 & AMPX & 06/08/01 & t108 & \\
\hline 28 & 35079 & br79 & 3525 & 35079 & ENDFB & V6 & RELO & REVO & MODO & AMPX & 06/08/01 & t102 & \\
\hline 29 & 35081 & br 81 & 3531 & 35081 & ENDFB & V6 & RELO & REVO & MOD0 & AMPX & 06/08/01 & t102 & \\
\hline 30 & 6012 & C & 600 & 6012 & endfb & v6 & relo & rev2 & $\bmod 3$ & ampx & 08/06/01 & rev6/tape. 145 & \\
\hline 31 & 20000 & $\mathrm{ca}$ & 2000 & 20000 & endfb & v6 & relo & rev1 & $\bmod 1$ & ampx & 08/06/01 & rev6/tape.146 & \\
\hline 32 & 48000 & $\mathrm{~cd}$ & 4800 & 48000 & endfb & v6 & relo & rev0 & $\bmod 0$ & ampx & 08/06/01 & t104 & \\
\hline 33 & 48106 & cd106 & 4825 & 48106 & ENDFB & V6 & RELO & REV2 & MOD4 & AMPX & 06/08/01 & rev4/tape.137 & \\
\hline 34 & 48108 & cd108 & 4831 & 48108 & ENDFB & V6 & RELO & REV2 & MOD4 & AMPX & $06 / 08 / 01$ & rev4/tape.137 & \\
\hline 35 & 48110 & cd110 & 4837 & 48110 & ENDFB & V6 & RELO & REV3 & MOD4 & AMPX & 06/08/01 & rev4/tape.137 & \\
\hline 36 & 48111 & $\operatorname{col} 111$ & 4840 & 48111 & ENDFB & V6 & RELO & REVO & MOD1 & AMPX & $06 / 08 / 01$ & rev3/tape.134 & \\
\hline 37 & 48112 & $\operatorname{cd} 112$ & 4843 & 48112 & ENDFB & V6 & RELO & REV2 & MOD4 & AMPX & $06 / 08 / 01$ & rev4/tape.137 & 2 \\
\hline 38 & 48113 & $\operatorname{col} 113$ & 4846 & 48113 & ENDFB & V6 & RELO & REVO & MOD1 & AMPX & $06 / 08 / 01$ & rev3/tape.134 & 2 \\
\hline 39 & 48114 & cd114 & 4849 & 48114 & ENDFB & V6 & RELO & REV2 & MOD4 & AMPX & $06 / 08 / 01$ & rev4/tape.137 & 2 \\
\hline 40 & 48601 & cd115m & 4853 & 48601 & endfb & v6 & relo & rev0 & $\bmod 0$ & ampx & 08/06/01 & t104 & 25 \\
\hline 41 & 48116 & cd116 & 4855 & 48116 & ENDFB & V6 & RELO & REV2 & MOD4 & AMPX & $06 / 08 / 01$ & rev4/tape.137 & 25 \\
\hline 42 & 58140 & ce140 & 5837 & 58140 & ENDFB & V6 & RELO & REVO & MOD0 & AMPX & 06/08/01 & t105 & 25 \\
\hline 43 & 58141 & ce141 & 5840 & 58141 & ENDFB & V6 & RELO & REVO & MODO & AMPX & $06 / 08 / 01$ & t105 & 25 \\
\hline 44 & 58142 & ce142 & 5843 & 58142 & ENDFB & V6 & RELO & REVO & MOD0 & AMPX & 06/08/01 & t105 & 25 \\
\hline 45 & 58143 & ce143 & 5846 & 58143 & ENDFB & V6 & RELO & REVO & MOD0 & AMPX & 06/08/01 & t105 & 25 \\
\hline 46 & 58144 & ce144 & 5849 & 58144 & ENDFB & V6 & RELO & REVO & MOD0 & AMPX & 06/08/01 & t105 & 25 \\
\hline 47 & 98249 & Cf249 & 9852 & 98249 & ENDFB & V6 & RELO & REVO & MOD1 & AMPX & 06/08/01 & t108 & 25 \\
\hline 48 & 98250 & Cf250 & 9855 & 98250 & ENDFB & V6 & RELO & REV1 & MOD2 & AMPX & 06/08/01 & rev2/tape.129 & \\
\hline 49 & 98251 & Cf251 & 9858 & 98251 & ENDFB & V6 & RELO & REV1 & MOD2 & AMPX & $06 / 08 / 01$ & rev2/tape.129 & 25 \\
\hline 50 & 98252 & cf252 & 9861 & 98252 & ENDFB & V6 & RELO & REV2 & MOD3 & AMPX & $06 / 08 / 01$ & rev2/tape.129 & 25 \\
\hline 51 & 98253 & cf253 & 9864 & 98253 & ENDFB & V6 & RELO & REV1 & MOD2 & AMPX & $06 / 08 / 01$ & rev2/tape.129 & 25 \\
\hline 52 & 17000 & cl & 1700 & 17000 & endfb & v6 & relo & rev0 & $\bmod 0$ & ampx & 08/06/01 & t101 & \\
\hline 53 & 96241 & $\mathrm{~cm} 241$ & 9628 & 96241 & ENDFB & V6 & RELO & REVO & MOD0 & AMPX & 06/08/01 & t110 & 2 \\
\hline 54 & 96242 & $\mathrm{~cm} 242$ & 9631 & 96242 & ENDFB & V6 & RELO & REVO & MOD1 & AMPX & 06/08/01 & t109 & \\
\hline 55 & 96243 & $\mathrm{~cm} 243$ & 9634 & 96243 & ENDFB & V6 & RELO & REVO & MOD1 & AMPX & 06/08/01 & rev7/tape.155 & 2 \\
\hline 56 & 96244 & $\mathrm{~cm} 244$ & 9637 & 96244 & ENDFB & V6 & RELO & REVO & MOD0 & AMPX & 06/08/01 & t110 & 25 \\
\hline 57 & 96245 & $\mathrm{~cm} 245$ & 9640 & 96245 & ENDFB & V6 & RELO & REVO & MOD3 & AMPX & $06 / 08 / 01$ & rev7/tape.155 & 25 \\
\hline 58 & 96246 & $\mathrm{~cm} 246$ & 9643 & 96246 & ENDFB & V6 & RELO & REVO & MOD3 & AMPX & $06 / 08 / 01$ & rev7/tape.155 & 2 \\
\hline 59 & 96247 & $\mathrm{~cm} 247$ & 9646 & 96247 & ENDFB & V6 & RELO & REV1 & MOD2 & AMPX & $06 / 08 / 01$ & rev2/tape.129 & 25 \\
\hline 60 & 96248 & $\mathrm{~cm} 248$ & 9649 & 96248 & ENDFB & V6 & RELO & REVO & MOD0 & AMPX & $06 / 08 / 01$ & t110 & 25 \\
\hline 61 & 27059 & co59 & 2725 & 27059 & ENDFB & V6 & RELO & REV1 & MOD2 & AMPX & $06 / 08 / 01$ & rev2/tape. 129 & \\
\hline
\end{tabular}




\begin{tabular}{|c|c|c|c|c|c|c|c|c|c|c|c|c|c|}
\hline Dentry & identifier & & & & & & $t i$ & $t 1 e$ & & & & & source \\
\hline 62 & 24050 & $\operatorname{cr} 50$ & 2425 & 24050 & ENDFB & V6 & RELO & REV3 & MOD4 & AMPX & $06 / 08 / 01$ & rev6/tape. 147 & 25 \\
\hline 63 & 24052 & $\operatorname{cr} 52$ & 2431 & 24052 & ENDFB & V6 & RELO & REV2 & MOD3 & AMPX & $06 / 08 / 01$ & rev6/tape. 147 & 25 \\
\hline 64 & 24053 & $\operatorname{cr} 53$ & 2434 & 24053 & ENDFB & V6 & RELO & REV2 & MOD3 & AMPX & $06 / 08 / 01$ & rev6/tape. 147 & \\
\hline 65 & 24054 & $\operatorname{cr} 54$ & 2437 & 24054 & ENDFB & V6 & RELO & REV3 & MOD4 & AMPX & $06 / 08 / 01$ & rev6/tape. 147 & \\
\hline 66 & 55133 & Cs133 & 5525 & 55133 & ENDFB & V6 & REL0 & REV0 & MOD1 & AMPX & $06 / 08 / 01$ & rev7/tape.154 & \\
\hline 67 & 55134 & Cs134 & 5528 & 55134 & ENDFB & V6 & REL0 & REV0 & MOD2 & AMPX & $06 / 08 / 01$ & rev7/tape.154 & \\
\hline 68 & 55135 & Cs135 & 5531 & 55135 & ENDFB & V6 & REL0 & REV0 & MOD1 & AMPX & $06 / 08 / 01$ & rev7/tape.154 & \\
\hline 69 & 55136 & Cs136 & 5534 & 55136 & ENDFB & V6 & RELO & REV0 & MOD0 & AMPX & 06/08/01 & t105 & \\
\hline 70 & 55137 & Cs137 & 5537 & 55137 & ENDFB & V6 & RELO & REVO & MOD0 & AMPX & $06 / 08 / 01$ & t105 & \\
\hline 71 & 29063 & cu63 & 2925 & 29063 & ENDFB & V6 & RELO & REV3 & MOD4 & AMPX & $06 / 08 / 01$ & rev6/tape. 150 & \\
\hline 72 & 29065 & cu65 & 2931 & 29065 & ENDFB & V6 & REL0 & REV3 & MOD4 & AMPX & $06 / 08 / 01$ & rev6/tape.150 & \\
\hline 73 & 1002 & d_d2o & 11 & 1002 & endfb & v6 & relo & rev0 & mod0 & ampx & 08/06/01 & t118 & \\
\hline 74 & 66160 & dy160 & 6637 & 66160 & ENDFB & V6 & RELO & REVO & MOD1 & AMPX & $06 / 08 / 01$ & rev7/tape.154 & \\
\hline 75 & 66161 & dy161 & 6640 & 66161 & ENDFB & V6 & RELO & REV0 & MOD1 & AMPX & 06/08/01 & rev7/tape.154 & \\
\hline 76 & 66162 & dy162 & 6643 & 66162 & ENDFB & V6 & RELO & REVO & MOD1 & AMPX & $06 / 08 / 01$ & rev7/tape. 154 & \\
\hline 77 & 66163 & dy163 & 6646 & 66163 & ENDFB & V6 & RELO & REVO & MOD1 & AMPX & $06 / 08 / 01$ & rev7/tape.154 & \\
\hline 78 & 66164 & dy164 & 6649 & 66164 & ENDFB & V6 & REL0 & REV0 & MOD1 & AMPX & $06 / 08 / 01$ & rev7/tape.154 & \\
\hline 79 & 68166 & er166 & 6837 & 68166 & ENDFB & V6 & RELO & REV0 & MOD1 & AMPX & $06 / 08 / 01$ & t103 & \\
\hline 80 & 68167 & er167 & 6840 & 68167 & ENDFB & V6 & RELO & REV0 & MOD1 & AMPX & $06 / 08 / 01$ & t103 & \\
\hline 81 & 99253 & es253 & 9913 & 99253 & ENDFB & V6 & RELO & REV0 & MOD0 & AMPX & $06 / 08 / 01$ & t110 & \\
\hline 82 & 63151 & eu151 & 6325 & 63151 & ENDFB & V6 & RELO & REV0 & MOD1 & AMPX & 06/08/01 & t103 & \\
\hline 83 & 63152 & eu152 & 6328 & 63152 & ENDFB & V6 & REL0 & REV0 & MOD1 & AMPX & $06 / 08 / 01$ & t103 & \\
\hline 84 & 63153 & eu153 & 6331 & 63153 & ENDFB & V6 & RELO & REV0 & MOD2 & AMPX & 06/08/01 & rev7/tape.154 & \\
\hline 85 & 63154 & eu154 & 6334 & 63154 & ENDFB & V6 & RELO & REV1 & MOD2 & AMPX & $06 / 08 / 01$ & rev7/tape.154 & \\
\hline 86 & 63155 & eu155 & 6337 & 63155 & ENDFB & V6 & RELO & REVO & MOD3 & AMPX & $06 / 08 / 01$ & rev7/tape.154 & \\
\hline 87 & 63156 & eu156 & 6340 & 63156 & ENDFB & V6 & RELO & REVO & MOD0 & AMPX & $06 / 08 / 01$ & t106 & \\
\hline 88 & 63157 & eu157 & 6343 & 63157 & ENDFB & V6 & RELO & REVO & MOD0 & AMPX & $06 / 08 / 01$ & t106 & \\
\hline 89 & 9019 & f19 & 925 & 9019 & ENDFB & V6 & RELO & REVO & MOD1 & AMPX & $06 / 08 / 01$ & t115 & \\
\hline 90 & 26054 & fe54 & 2625 & 26054 & ENDFB & V6 & RELO & REV3 & MOD5 & AMPX & 06/08/01 & rev6/tape.148 & \\
\hline 91 & 26056 & fe56 & 2631 & 26056 & ENDFB & V6 & RELO & REV2 & MOD3 & AMPX & $06 / 08 / 01$ & rev6/tape.148 & \\
\hline 92 & 26057 & fe57 & 2634 & 26057 & ENDFB & V6 & RELO & REV2 & MOD3 & AMPX & $06 / 08 / 01$ & rev6/tape.148 & \\
\hline 93 & 26058 & fe58 & 2637 & 26058 & ENDFB & V6 & REL0 & REV2 & MOD3 & AMPX & $06 / 08 / 01$ & rev5/tape.140 & \\
\hline 94 & 31000 & ga & 3100 & 31000 & endfb & v6 & relo & rev0 & mod0 & ampx & 08/06/01 & t102 & \\
\hline 95 & 64152 & gd152 & 6425 & 64152 & ENDFB & V6 & RELO & REVO & MOD1 & AMPX & $06 / 08 / 01$ & rev4/tape.137 & \\
\hline 96 & 64154 & gd154 & 6431 & 64154 & ENDFB & V6 & RELO & REV0 & MOD1 & AMPX & $06 / 08 / 01$ & rev4/tape.137 & \\
\hline 97 & 64155 & gd155 & 6434 & 64155 & ENDFB & V6 & RELO & REVO & MOD0 & AMPX & $06 / 08 / 01$ & t106 & \\
\hline 98 & 64156 & gd156 & 6437 & 64156 & ENDFB & V6 & RELO & REVO & MOD0 & AMPX & $06 / 08 / 01$ & t106 & \\
\hline 99 & 64157 & gd157 & 6440 & 64157 & ENDFB & V6 & RELO & REV0 & MOD0 & AMPX & $06 / 08 / 01$ & t106 & \\
\hline 100 & 64158 & gd158 & 6443 & 64158 & ENDFB & V6 & RELO & REVO & MOD0 & AMPX & $06 / 08 / 01$ & t106 & \\
\hline 101 & 64160 & gd160 & 6449 & 64160 & ENDFB & V6 & RELO & REV0 & MOD0 & AMPX & $06 / 08 / 01$ & t106 & \\
\hline 102 & 32072 & ge72 & 3231 & 32072 & ENDFB & V6 & REL0 & REV0 & MOD0 & AMPX & 06/08/01 & t102 & \\
\hline 103 & 32073 & ge73 & 3234 & 32073 & ENDFB & V6 & REL0 & REV1 & MOD2 & AMPX & $06 / 08 / 01$ & rev2/tape.129 & \\
\hline 104 & 32074 & ge74 & 3237 & 32074 & ENDFB & V6 & REL0 & REV0 & MOD0 & AMPX & $06 / 08 / 01$ & t102 & \\
\hline 105 & 32076 & ge76 & 3243 & 32076 & ENDFB & V6 & RELO & REV0 & MOD0 & AMPX & 06/08/01 & t102 & \\
\hline 106 & 6312 & graphite & 31 & 6312 & endfb & v6 & relo & rev0 & mod1 & ampx & $08 / 06 / 01$ & rev3/tape.133 & \\
\hline 107 & 1901 & h_ch2 & 37 & 1901 & endfb & v6 & relo & rev0 & $\bmod 0$ & ampx & $08 / 06 / 01$ & t118 & \\
\hline 108 & 1001 & h_h2o & 1 & 1001 & endfb & v6 & relo & rev0 & $\bmod 1$ & ampx & $08 / 06 / 01$ & rev3/tape. 132 & \\
\hline 109 & 1701 & h_zrh & 7 & 1701 & endfb & v6 & relo & rev0 & $\bmod 1$ & ampx & $08 / 06 / 01$ & rev3/tape. 132 & \\
\hline 110 & 1801 & h1 & 125 & 1801 & endfb & v6 & relo & rev3 & $\bmod 4$ & ampx & $08 / 06 / 01$ & rev6/tape. 145 & \\
\hline 111 & 1802 & h2 & 128 & 1802 & endfb & v6 & relo & rev3 & $\bmod 4$ & ampx & $08 / 06 / 01$ & rev6/tape. 145 & \\
\hline 112 & 2003 & he3 & 225 & 2003 & ENDFB & V6 & RELO & REV1 & MOD2 & AMPX & $06 / 08 / 01$ & t120 & \\
\hline 113 & 2004 & he4 & 228 & 2004 & ENDFB & V6 & RELO & REVO & MOD0 & AMPX & $06 / 08 / 01$ & t101 & \\
\hline 114 & 72000 & hf & 7200 & 72000 & endfb & v6 & relo & rev0 & mod0 & ampx & $08 / 06 / 01$ & t106 & \\
\hline 115 & 72174 & hf174 & 7225 & 72174 & ENDFB & V6 & RELO & REV1 & MOD2 & AMPX & $06 / 08 / 01$ & rev2/tape.127 & \\
\hline 116 & 72176 & hf176 & 7231 & 72176 & ENDFB & V6 & RELO & REV1 & MOD2 & AMPX & $06 / 08 / 01$ & rev2 / tape. 127 & \\
\hline 117 & 72177 & hf177 & 7234 & 72177 & ENDFB & V6 & RELO & REV1 & MOD2 & AMPX & $06 / 08 / 01$ & rev2/tape.127 & \\
\hline 118 & 72178 & hf178 & 7237 & 72178 & ENDFB & V6 & RELO & REV1 & MOD2 & AMPX & $06 / 08 / 01$ & rev2/tape. 127 & \\
\hline 119 & 72179 & hf179 & 7240 & 72179 & ENDFB & V6 & REL0 & REV1 & MOD2 & AMPX & $06 / 08 / 01$ & rev2/tape.127 & \\
\hline 120 & 72180 & hf180 & 7243 & 72180 & ENDFB & V6 & RELO & REV1 & MOD2 & AMPX & $06 / 08 / 01$ & rev2 / tape. 127 & \\
\hline 121 & 67165 & ho165 & 6725 & 67165 & ENDFB & V6 & RELO & REV1 & MOD2 & AMPX & $06 / 08 / 01$ & rev5/tape. 143 & \\
\hline 122 & 53127 & i127 & 5325 & 53127 & ENDFB & V6 & RELO & REVO & MOD1 & AMPX & $06 / 08 / 01$ & rev2 / tape. 127 & \\
\hline 123 & 53129 & i129 & 5331 & 53129 & ENDFB & V6 & REL0 & REV0 & MOD0 & AMPX & 06/08/01 & t105 & \\
\hline 124 & 53130 & i130 & 5334 & 53130 & ENDFB & V6 & REL0 & REV0 & MOD0 & AMPX & $06 / 08 / 01$ & t105 & \\
\hline 125 & 53131 & i131 & 5337 & 53131 & ENDFB & V6 & REL0 & REV0 & MOD0 & AMPX & $06 / 08 / 01$ & t105 & \\
\hline 126 & 53135 & i135 & 5349 & 53135 & ENDFB & V6 & RELO & REV0 & MOD0 & AMPX & 06/08/01 & t105 & \\
\hline 127 & 49000 & in & 4900 & 49000 & endfb & v6 & relo & rev0 & mod1 & ampx & 08/06/01 & t116 & \\
\hline 128 & 49113 & in113 & 4925 & 49113 & ENDFB & V6 & RELO & REVO & MOD0 & AMPX & $06 / 08 / 01$ & t116 & \\
\hline 129 & 49115 & in115 & 4931 & 49115 & ENDFB & V6 & RELO & REVO & MOD1 & AMPXI & LNCOMPLETE & t116 & \\
\hline 130 & 77191 & ir191 & 7725 & 77191 & ENDFB & V6 & REL0 & REV0 & MOD1 & AMPX & $06 / 08 / 01$ & rev4/tape.137 & \\
\hline 131 & 77193 & ir193 & 7731 & 77193 & ENDFB & V6 & RELO & REV0 & MOD1 & AMPX & $06 / 08 / 01$ & rev4/tape.137 & \\
\hline 132 & 19000 & $\mathrm{k}$ & 1900 & 19000 & endfb & v6 & relo & rev0 & mod0 & ampx & 08/06/01 & t101 & \\
\hline 133 & 19041 & k41 & 1931 & 19041 & ENDFB & V6 & RELO & REVO & MOD0 & AMPXI & LNCOMPLETE & t101 & \\
\hline 134 & 36078 & $\mathrm{kr} 78$ & 3625 & 36078 & ENDFB & V6 & RELO & REVO & MOD0 & AMPX & $06 / 08 / 01$ & t102 & \\
\hline 135 & 36080 & $\mathrm{kr} 80$ & 3631 & 36080 & ENDFB & V6 & RELO & REV0 & MOD0 & AMPX & $06 / 08 / 01$ & t102 & \\
\hline 136 & 36082 & $\mathrm{kr} 82$ & 3637 & 36082 & ENDFB & V6 & RELO & REVO & MOD0 & AMPX & $06 / 08 / 01$ & t102 & \\
\hline 137 & 36083 & $\mathrm{kr} 83$ & 3640 & 36083 & ENDFB & V6 & RELO & REV0 & MOD0 & AMPX & $06 / 08 / 01$ & t102 & \\
\hline 138 & 36084 & $\mathrm{kr} 84$ & 3643 & 36084 & ENDFB & V6 & RELO & REV0 & MOD0 & AMPX & 06/08/01 & t102 & \\
\hline 139 & 36085 & kr85 & 3646 & 36085 & ENDFB & V6 & REL0 & REV0 & MOD0 & AMPX & $06 / 08 / 01$ & t102 & \\
\hline 140 & 36086 & $\operatorname{kr} 86$ & 3649 & 36086 & ENDFB & V6 & REL0 & REV0 & MOD0 & AMPX & $06 / 08 / 01$ & t102 & \\
\hline
\end{tabular}




\begin{tabular}{|c|c|c|c|c|c|c|c|c|c|c|c|c|c|}
\hline Dentry & identifier & \multicolumn{11}{|c|}{ t i t $1 \mathrm{e}$} & source \\
\hline 141 & 57139 & la139 & 5728 & 57139 & ENDFB & V6 & RELO & REV1 & MOD2 & AMPX & 06/08/01 & t120 & 25 \\
\hline 142 & 57140 & 1 a140 & 5731 & 57140 & ENDFB & V6 & RELO & REVO & MOD0 & AMPX & $06 / 08 / 01$ & t105 & 25 \\
\hline 143 & 1101 & lch4 & 33 & 1101 & endfb & v6 & relo & rev0 & $\bmod 1$ & ampx & $08 / 06 / 01$ & rev3/tape. 133 & \\
\hline 144 & 3006 & $1 i 6$ & 325 & 3006 & ENDFB & V6 & RELO & REV1 & MOD2 & AMPX & $06 / 08 / 01$ & t120 & \\
\hline 145 & 3007 & $1 i 7$ & 328 & 3007 & ENDFB & V6 & RELO & REVO & MOD1 & AMPX & $06 / 08 / 01$ & t100 & \\
\hline 146 & 71175 & lu175 & 7125 & 71175 & ENDFB & V6 & RELO & REV1 & MOD1 & AMPX & $06 / 08 / 01$ & rev7/tape. 154 & \\
\hline 147 & 71176 & $\operatorname{lu} 176$ & 7128 & 71176 & ENDFB & V6 & RELO & REV1 & MOD1 & AMPX & $06 / 08 / 01$ & rev7/tape. 154 & \\
\hline 148 & 12000 & $\mathrm{mg}$ & 1200 & 12000 & endfb & v6 & relo & rev0 & $\bmod 0$ & ampx & $08 / 06 / 01$ & t101 & \\
\hline 149 & 12024 & mg24 & 1225 & 12024 & ENDFB & V6 & RELO & REVO & MOD0 & AMPXI & LNCOMPLETE & t 101 & \\
\hline 150 & 25055 & mn55 & 2525 & 25055 & ENDFB & V6 & RELO & REV1 & MOD2 & AMPX & $06 / 08 / 01$ & rev5/tape. 140 & \\
\hline 151 & 42000 & mo & 4200 & 42000 & endfb & v6 & relo & rev0 & $\bmod 0$ & ampx & $08 / 06 / 01$ & t104 & \\
\hline 152 & 42100 & mo100 & 4249 & 42100 & ENDFB & V6 & RELO & REVO & MODO & AMPXI & CNCOMPLETE & t104 & \\
\hline 153 & 42092 & mo92 & 4225 & 42092 & ENDFB & V6 & RELO & REVO & MOD0 & AMPXI & CNCOMPLETE & t104 & \\
\hline 154 & 42094 & $\operatorname{mog} 4$ & 4231 & 42094 & ENDFB & V6 & RELO & REVO & MOD0 & AMPX & $06 / 08 / 01$ & t104 & \\
\hline 155 & 42095 & mo95 & 4234 & 42095 & ENDFB & V6 & RELO & REVO & MOD0 & AMPX & $06 / 08 / 01$ & t104 & \\
\hline 156 & 42096 & mo96 & 4237 & 42096 & ENDFB & V6 & RELO & REVO & MOD0 & AMPX & $06 / 08 / 01$ & t104 & \\
\hline 157 & 42097 & $\operatorname{mog} 7$ & 4240 & 42097 & ENDFB & V6 & RELO & REVO & MOD0 & AMPX & $06 / 08 / 01$ & t104 & \\
\hline 158 & 42098 & $\operatorname{mog} 8$ & 4243 & 42098 & ENDFB & V6 & RELO & REVO & MOD0 & AMPXI & LNCOMPLETE & t 104 & \\
\hline 159 & 42099 & mo99 & 4246 & 42099 & ENDFB & V6 & RELO & REVO & MOD0 & AMPX & $06 / 08 / 01$ & t104 & \\
\hline 160 & 7014 & n14 & 725 & 7014 & ENDFB & V6 & RELO & REV3 & MOD4 & AMPX & $06 / 08 / 01$ & rev6/tape. 145 & \\
\hline 161 & 7015 & n15 & 728 & 7015 & ENDFB & V6 & RELO & REVO & MOD1 & AMPX & $06 / 08 / 01$ & t116 & \\
\hline 162 & 11023 & na23 & 1125 & 11023 & ENDFB & V6 & RELO & REV1 & MOD2 & AMPX & $06 / 08 / 01$ & t120 & \\
\hline 163 & 41093 & nb93 & 4125 & 41093 & ENDFB & V6 & RELO & REV2 & MOD3 & AMPX & $06 / 08 / 01$ & rev6/tape. 150 & \\
\hline 164 & 41094 & nb94 & 4128 & 41094 & ENDFB & V6 & RELO & REVO & MODO & AMPX & $06 / 08 / 01$ & t104 & \\
\hline 165 & 41095 & nb95 & 4131 & 41095 & ENDFB & V6 & RELO & REVO & MOD0 & AMPX & $06 / 08 / 01$ & t104 & \\
\hline 166 & 60142 & nd142 & 6025 & 60142 & ENDFB & V6 & RELO & REVO & MOD0 & AMPX & $06 / 08 / 01$ & t105 & \\
\hline 167 & 60143 & nd143 & 6028 & 60143 & ENDFB & V6 & RELO & REV1 & MOD2 & AMPX & $06 / 08 / 01$ & rev2 / tape. 127 & \\
\hline 168 & 60144 & nd144 & 6031 & 60144 & ENDFB & V6 & RELO & REVO & MOD0 & AMPX & $06 / 08 / 01$ & t105 & \\
\hline 169 & 60145 & nd145 & 6034 & 60145 & ENDFB & V6 & RELO & REV1 & MOD2 & AMPX & $06 / 08 / 01$ & rev2/tape. 127 & \\
\hline 170 & 60146 & nd146 & 6037 & 60146 & ENDFB & V6 & RELO & REVO & MOD0 & AMPX & $06 / 08 / 01$ & t105 & \\
\hline 171 & 60147 & nd147 & 6040 & 60147 & ENDFB & V6 & RELO & REV1 & MOD2 & AMPX & $06 / 08 / 01$ & t120 & \\
\hline 172 & 60148 & nd148 & 6043 & 60148 & ENDFB & V6 & RELO & REVO & MOD0 & AMPX & $06 / 08 / 01$ & t105 & \\
\hline 173 & 60150 & nd150 & 6049 & 60150 & ENDFB & V6 & RELO & REVO & MOD0 & AMPX & $06 / 08 / 01$ & t105 & \\
\hline 174 & 28058 & ni58 & 2825 & 28058 & ENDFB & V6 & REL0 & REV2 & MOD3 & AMPX & $06 / 08 / 01$ & rev6/tape.149 & \\
\hline 175 & 28059 & ni59 & 2828 & 28059 & ENDFB & V6 & RELO & REVO & MOD1 & AMPXI & CNCOMPLETE & t113 & \\
\hline 176 & 28060 & ni60 & 2831 & 28060 & ENDFB & V6 & RELO & REV2 & MOD3 & AMPX & $06 / 08 / 01$ & rev6/tape. 149 & \\
\hline 177 & 28061 & ni61 & 2834 & 28061 & ENDFB & V6 & RELO & REV3 & MOD4 & AMPX & $06 / 08 / 01$ & rev6/tape. 149 & \\
\hline 178 & 28062 & ni62 & 2837 & 28062 & ENDFB & V6 & RELO & REV3 & MOD4 & AMPX & $06 / 08 / 01$ & rev6/tape. 149 & \\
\hline 179 & 28064 & ni64 & 2843 & 28064 & ENDFB & V6 & REL0 & REV2 & MOD3 & AMPX & $06 / 08 / 01$ & rev6/tape. 149 & \\
\hline 180 & 93237 & np237 & 9346 & 93237 & ENDFB & V6 & RELO & REV1 & MOD2 & AMPX & $06 / 08 / 01$ & t121 & \\
\hline 181 & 93238 & np238 & 9349 & 93238 & ENDFB & V6 & RELO & REV1 & MOD2 & AMPX & $06 / 08 / 01$ & rev2/tape. 129 & \\
\hline 182 & 93239 & np239 & 9352 & 93239 & ENDFB & V6 & RELO & REVO & MOD1 & AMPX & $06 / 08 / 01$ & t108 & \\
\hline 183 & 8016 & 016 & 825 & 8016 & ENDFB & V6 & RELO & REV1 & MOD2 & AMPX & $06 / 08 / 01$ & rev6/tape. 145 & \\
\hline 184 & 8017 & o17 & 828 & 8017 & ENDFB & V6 & RELO & REVO & MOD0 & AMPX & $06 / 08 / 01$ & t101 & \\
\hline 185 & 1402 & orthod & 13 & 1402 & endfb & v6 & relo & rev0 & $\operatorname{mod1}$ & ampx & $08 / 06 / 01$ & rev3/tape. 132 & \\
\hline 186 & 1401 & orthoh & 3 & 1401 & endfb & v6 & relo & rev0 & $\bmod 1$ & ampx & $08 / 06 / 01$ & rev3/tape. 132 & \\
\hline 187 & 15031 & p31 & 1525 & 15031 & ENDFB & V6 & RELO & REV1 & MOD1 & AMPX & $06 / 08 / 01$ & rev6/tape. 146 & \\
\hline 188 & 91231 & pa231 & 9131 & 91231 & ENDFB & V6 & RELO & REVO & MOD1 & AMPX & $06 / 08 / 01$ & t109 & \\
\hline 189 & 91233 & pa233 & 9137 & 91233 & ENDFB & V6 & RELO & REVO & MOD0 & AMPX & $06 / 08 / 01$ & t110 & \\
\hline 190 & 1502 & parad & 12 & 1502 & endfb & v6 & relo & rev0 & $\bmod 1$ & ampx & $08 / 06 / 01$ & rev3/tape. 132 & \\
\hline 191 & 1501 & parah & 2 & 1501 & endfb & v6 & relo & rev0 & $\operatorname{mod1}$ & ampx & $08 / 06 / 01$ & rev3/tape. 132 & \\
\hline 192 & 82206 & pb206 & 8231 & 82206 & ENDFB & V6 & RELO & REV1 & MOD2 & AMPX & $06 / 08 / 01$ & rev6/tape. 152 & \\
\hline 193 & 82207 & pb207 & 8234 & 82207 & ENDFB & V6 & RELO & REV2 & MOD3 & AMPX & $06 / 08 / 01$ & rev6/tape. 152 & \\
\hline 194 & 82208 & pb208 & 8237 & 82208 & ENDFB & V6 & REL0 & REV2 & MOD3 & AMPX & $06 / 08 / 01$ & rev6/tape.152 & \\
\hline 195 & 46102 & pd102 & 4625 & 46102 & ENDFB & V6 & REL0 & REVO & MOD1 & AMPX & $06 / 08 / 01$ & rev5/tape.141 & \\
\hline 196 & 46104 & pd104 & 4631 & 46104 & ENDFB & V6 & REL0 & REVO & MOD1 & AMPX & $06 / 08 / 01$ & rev5/tape.141 & \\
\hline 197 & 46105 & pd105 & 4634 & 46105 & ENDFB & V6 & REL0 & REVO & MOD2 & AMPX & $06 / 08 / 01$ & rev5/tape.141 & \\
\hline 198 & 46106 & pd106 & 4637 & 46106 & ENDFB & V6 & RELO & REVO & MOD1 & AMPX & $06 / 08 / 01$ & rev5/tape. 141 & \\
\hline 199 & 46107 & pd107 & 4640 & 46107 & ENDFB & V6 & RELO & REVO & MOD1 & AMPX & $06 / 08 / 01$ & t103 & \\
\hline 200 & 46108 & pd108 & 4643 & 46108 & ENDFB & V6 & RELO & REVO & MOD1 & AMPX & $06 / 08 / 01$ & rev5/tape. 141 & \\
\hline 201 & 46110 & pd110 & 4649 & 46110 & ENDFB & V6 & RELO & REVO & MOD1 & AMPX & $06 / 08 / 01$ & rev5/tape. 141 & \\
\hline 202 & 61147 & pm147 & 6149 & 61147 & ENDFB & V6 & RELO & REV1 & MOD2 & AMPX & $06 / 08 / 01$ & t120 & \\
\hline 203 & 61148 & pm148 & 6152 & 61148 & ENDFB & V6 & RELO & REVO & MOD0 & AMPX & $06 / 08 / 01$ & t106 & \\
\hline 204 & 61601 & pm148m & 6153 & 61601 & endfb & v6 & relo & rev0 & modo & ampx & $08 / 06 / 01$ & t106 & \\
\hline 205 & 61149 & pm149 & 6155 & 61149 & ENDFB & V6 & RELO & REVO & MOD0 & AMPX & $06 / 08 / 01$ & t106 & \\
\hline 206 & 61151 & pm151 & 6161 & 61151 & ENDFB & V6 & RELO & REVO & MOD0 & AMPX & $06 / 08 / 01$ & t106 & \\
\hline 207 & 59141 & pr141 & 5925 & 59141 & ENDFB & V6 & RELO & REVO & MOD1 & AMPX & $06 / 08 / 01$ & rev7/tape. 154 & \\
\hline 208 & 59142 & pr142 & 5928 & 59142 & ENDFB & V6 & RELO & REVO & MOD0 & AMPX & $06 / 08 / 01$ & t105 & \\
\hline 209 & 59143 & pr143 & 5931 & 59143 & ENDFB & V6 & RELO & REVO & MOD0 & AMPX & $06 / 08 / 01$ & t105 & \\
\hline 210 & 94236 & pu236 & 9428 & 94236 & ENDFB & V6 & RELO & REVO & MOD1 & AMPX & $06 / 08 / 01$ & rev4/tape .137 & \\
\hline 211 & 94237 & pu237 & 9431 & 94237 & ENDFB & V6 & REL0 & REV0 & MOD0 & AMPX & $06 / 08 / 01$ & t110 & \\
\hline 212 & 94238 & pu238 & 9434 & 94238 & ENDFB & V6 & RELO & REVO & MOD1 & AMPX & $06 / 08 / 01$ & t109 & \\
\hline 213 & 94239 & pu239 & 9437 & 94239 & ENDFB & V6 & RELO & REV2 & MOD3 & AMPX & $06 / 08 / 01$ & rev5/tape. 142 & \\
\hline 214 & 94240 & pu240 & 9440 & 94240 & ENDFB & V6 & RELO & REV2 & MOD3 & AMPX & $06 / 08 / 01$ & rev2/tape. 128 & \\
\hline 215 & 94241 & pu241 & 9443 & 94241 & ENDFB & V6 & RELO & REV2 & MOD3 & AMPX & $06 / 08 / 01$ & rev3/tape.135 & \\
\hline 216 & 94242 & pu242 & 9446 & 94242 & ENDFB & V6 & REL0 & REVO & MOD1 & AMPX & $06 / 08 / 01$ & t109 & \\
\hline 217 & 94243 & pu243 & 9449 & 94243 & ENDFB & V6 & REL0 & REV1 & MOD2 & AMPX & $06 / 08 / 01$ & rev2/tape. 129 & \\
\hline 218 & 94244 & pu244 & 9452 & 94244 & ENDFB & V6 & REL0 & REVO & MOD0 & AMPX & $06 / 08 / 01$ & t110 & \\
\hline 219 & 37085 & rb85 & 3725 & 37085 & ENDFB & V6 & RELO & REVO & MOD0 & AMPX & $06 / 08 / 01$ & t102 & \\
\hline
\end{tabular}




\begin{tabular}{|c|c|c|c|c|c|c|c|c|c|c|c|c|c|}
\hline Oentry & identifier & \multicolumn{11}{|c|}{ t i t 1 e } & sourc \\
\hline 220 & 37086 & rb86 & 3728 & 37086 & ENDFB & V6 & RELO & REVO & MODO & AMPX & $06 / 08 / 01$ & t102 & \\
\hline 221 & 37087 & rb87 & 3731 & 37087 & ENDFB & V6 & RELO & REVO & MOD0 & AMPX & $06 / 08 / 01$ & t102 & \\
\hline 222 & 75185 & re185 & 7525 & 75185 & ENDFB & V6 & RELO & REVO & MOD1 & AMPX & $06 / 08 / 01$ & t115 & \\
\hline 223 & 75187 & re187 & 7531 & 75187 & ENDFB & V6 & RELO & REVO & MOD1 & AMPX & $06 / 08 / 01$ & t115 & \\
\hline 224 & 45103 & rh103 & 4525 & 45103 & ENDFB & V6 & RELO & REVO & MODO & AMPX & $06 / 08 / 01$ & t104 & \\
\hline 225 & 45105 & rh105 & 4531 & 45105 & ENDFB & V6 & RELO & REVO & MODO & AMPX & $06 / 08 / 01$ & t104 & \\
\hline 226 & 44100 & ru100 & 4437 & 44100 & ENDFB & V6 & RELO & REVO & MODO & AMPX & 06/08/01 & t104 & \\
\hline 227 & 44101 & ru101 & 4440 & 44101 & ENDFB & V6 & RELO & REV1 & MOD2 & AMPX & $06 / 08 / 01$ & rev2/tape.127 & \\
\hline 228 & 44102 & ru102 & 4443 & 44102 & ENDFB & V6 & RELO & REV1 & MOD2 & AMPX & $06 / 08 / 01$ & rev2/tape. 127 & \\
\hline 229 & 44103 & ru103 & 4446 & 44103 & ENDFB & V6 & RELO & REVO & MODO & AMPX & $06 / 08 / 01$ & t104 & \\
\hline 230 & 44104 & ru104 & 4449 & 44104 & ENDFB & V6 & RELO & REVO & MODO & AMPX & $06 / 08 / 01$ & t104 & \\
\hline 231 & 44105 & ru105 & 4452 & 44105 & ENDFB & V6 & RELO & REVO & MOD0 & AMPX & $06 / 08 / 01$ & t104 & \\
\hline 232 & 44106 & ru106 & 4455 & 44106 & ENDFB & V6 & RELO & REVO & MODO & AMPX & $06 / 08 / 01$ & t104 & \\
\hline 233 & 44096 & ru96 & 4425 & 44096 & ENDFB & V6 & RELO & REVO & MODO & AMPX & $06 / 08 / 01$ & t104 & \\
\hline 234 & 44098 & ru98 & 4431 & 44098 & ENDFB & V6 & RELO & REVO & MODO & AMPX & $06 / 08 / 01$ & t104 & \\
\hline 235 & 44099 & ru99 & 4434 & 44099 & ENDFB & V6 & RELO & REVO & MODO & AMPX & $06 / 08 / 01$ & t104 & \\
\hline 236 & 16000 & $\mathrm{~s}$ & 1600 & 16000 & endfb & v6 & relo & rev0 & $\bmod 0$ & ampx & 08/06/01 & t101 & \\
\hline 237 & 16032 & s32 & 1625 & 16032 & ENDFB & V6 & RELO & REVO & MODO & AMPX & $06 / 08 / 01$ & t101 & \\
\hline 238 & 51121 & $\mathrm{sb} 121$ & 5125 & 51121 & ENDFB & V6 & RELO & REVO & MOD0 & AMPX & 06/08/01 & t105 & \\
\hline 239 & 51123 & sb123 & 5131 & 51123 & ENDFB & V6 & RELO & REVO & MODO & AMPX & $06 / 08 / 01$ & t105 & \\
\hline 240 & 51124 & $\mathrm{sb} 124$ & 5134 & 51124 & ENDFB & V6 & RELO & REVO & MODO & AMPX & 06/08/01 & t105 & \\
\hline 241 & 51125 & sb125 & 5137 & 51125 & ENDFB & V6 & RELO & REVO & MODO & AMPX & 06/08/01 & t105 & \\
\hline 242 & 51126 & sb126 & 5140 & 51126 & ENDFB & V6 & RELO & REVO & MODO & AMPX & $06 / 08 / 01$ & t105 & \\
\hline 243 & 21045 & $\mathrm{sc} 45$ & 2125 & 21045 & ENDFB & V6 & RELO & REVO & MOD1 & AMPX & $06 / 08 / 01$ & rev2/tape. 127 & \\
\hline 244 & 1201 & sch 4 & 34 & 1201 & endfb & v6 & relo & rev0 & $\bmod 1$ & ampx & $08 / 06 / 01$ & rev3/tape.133 & \\
\hline 245 & 34074 & se74 & 3425 & 34074 & ENDFB & V6 & RELO & REVO & MOD0 & AMPX & $06 / 08 / 01$ & t102 & \\
\hline 246 & 34076 & se76 & 3431 & 34076 & ENDFB & V6 & RELO & REVO & MOD0 & AMPX & $06 / 08 / 01$ & t102 & \\
\hline 247 & 34077 & se77 & 3434 & 34077 & ENDFB & V6 & RELO & REVO & MODO & AMPX & 06/08/01 & t102 & \\
\hline 248 & 34078 & se78 & 3437 & 34078 & ENDFB & V6 & RELO & REVO & MODO & AMPX & $06 / 08 / 01$ & t102 & \\
\hline 249 & 34080 & se 80 & 3443 & 34080 & ENDFB & V6 & RELO & REVO & MODO & AMPX & $06 / 08 / 01$ & t102 & \\
\hline 250 & 34082 & se82 & 3449 & 34082 & ENDFB & V6 & RELO & REVO & MODO & AMPX & $06 / 08 / 01$ & t102 & \\
\hline 251 & 14000 & si & 1400 & 14000 & endfb & v6 & relo & rev0 & $\bmod 0$ & ampx & 08/06/01 & t116 & \\
\hline 252 & 14028 & $\operatorname{si28}$ & 1425 & 14028 & ENDFB & V6 & RELO & REVO & MOD3 & AMPX & $06 / 08 / 01$ & rev5/tape.139 & \\
\hline 253 & 14029 & si29 & 1428 & 14029 & ENDFB & V6 & RELO & REV1 & MOD2 & AMPX & $06 / 08 / 01$ & rev6/tape. 146 & \\
\hline 254 & 14030 & $\operatorname{si30}$ & 1431 & 14030 & ENDFB & V6 & RELO & REV1 & MOD2 & AMPX & $06 / 08 / 01$ & rev6/tape. 146 & \\
\hline 255 & 62144 & $\operatorname{sm} 144$ & 6225 & 62144 & ENDFB & V6 & RELO & REV1 & MOD2 & AMPX & $06 / 08 / 01$ & rev3/tape. 134 & \\
\hline 256 & 62147 & $\operatorname{sm147}$ & 6234 & 62147 & ENDFB & V6 & RELO & REVO & MOD1 & AMPX & $06 / 08 / 01$ & t103 & \\
\hline 257 & 62148 & $\operatorname{sm148}$ & 6237 & 62148 & ENDFB & V6 & RELO & REVO & MODO & AMPX & 06/08/01 & t106 & \\
\hline 258 & 62149 & $\operatorname{sm149}$ & 6240 & 62149 & ENDFB & V6 & RELO & REVO & MOD1 & AMPX & $06 / 08 / 01$ & rev7/tape. 154 & \\
\hline 259 & 62150 & sm150 & 6243 & 62150 & ENDFB & V6 & RELO & REVO & MOD2 & AMPX & $06 / 08 / 01$ & rev2/tape.127 & \\
\hline 260 & 62151 & sm151 & 6246 & 62151 & ENDFB & V6 & RELO & REV1 & MOD2 & AMPX & $06 / 08 / 01$ & t120 & \\
\hline 261 & 62152 & sm152 & 6249 & 62152 & ENDFB & V6 & RELO & REV1 & MOD2 & AMPX & $06 / 08 / 01$ & rev2/tape. 127 & \\
\hline 262 & 62153 & sm153 & 6252 & 62153 & ENDFB & V6 & RELO & REVO & MODO & AMPX & $06 / 08 / 01$ & t106 & \\
\hline 263 & 62154 & $\operatorname{sm154}$ & 6255 & 62154 & ENDFB & V6 & RELO & REVO & MODO & AMPX & $06 / 08 / 01$ & t106 & \\
\hline 264 & 50112 & $\operatorname{sn} 112$ & 5025 & 50112 & ENDFB & V6 & RELO & REV1 & MOD2 & AMPX & $06 / 08 / 01$ & t120 & \\
\hline 265 & 50114 & $\operatorname{sn} 114$ & 5031 & 50114 & ENDFB & V6 & RELO & REV1 & MOD2 & AMPX & $06 / 08 / 01$ & t120 & \\
\hline 266 & 50115 & sn115 & 5034 & 50115 & ENDFB & V6 & RELO & REVO & MOD0 & AMPX & $06 / 08 / 01$ & t105 & \\
\hline 267 & 50116 & $\operatorname{sn} 116$ & 5037 & 50116 & ENDFB & V6 & RELO & REVO & MOD0 & AMPX & $06 / 08 / 01$ & t105 & \\
\hline 268 & 50117 & $\operatorname{sn} 117$ & 5040 & 50117 & ENDFB & V6 & RELO & REVO & MODO & AMPX & 06/08/01 & t105 & \\
\hline 269 & 50118 & $\operatorname{sn} 118$ & 5043 & 50118 & ENDFB & V6 & RELO & REVO & MODO & AMPX & 06/08/01 & t105 & \\
\hline 270 & 50119 & $\operatorname{sn} 119$ & 5046 & 50119 & ENDFB & V6 & RELO & REVO & MODO & AMPX & 06/08/01 & t105 & \\
\hline 271 & 50120 & $\operatorname{sn} 120$ & 5049 & 50120 & ENDFB & V6 & RELO & REVO & MODO & AMPXI & INCOMPLETE & t 105 & \\
\hline 272 & 50122 & $\operatorname{sn} 122$ & 5055 & 50122 & ENDFB & V6 & RELO & REVO & MODO & AMPXI & INCOMPLETE & t105 & \\
\hline 273 & 50123 & $\operatorname{sn} 123$ & 5058 & 50123 & ENDFB & V6 & RELO & REVO & MODO & AMPX & $06 / 08 / 01$ & t105 & \\
\hline 274 & 50124 & $\operatorname{sn} 124$ & 5061 & 50124 & ENDFB & V6 & RELO & REVO & MODO & AMPXI & INCOMPLETE & t105 & \\
\hline 275 & 50125 & $\operatorname{sn} 125$ & 5064 & 50125 & ENDFB & V6 & RELO & REVO & MODO & AMPX & $06 / 08 / 01$ & t105 & \\
\hline 276 & 50126 & $\operatorname{sn} 126$ & 5067 & 50126 & ENDFB & V6 & RELO & REVO & MODO & AMPX & $06 / 08 / 01$ & t105 & \\
\hline 277 & 38084 & $\operatorname{sr} 84$ & 3825 & 38084 & ENDFB & V6 & RELO & REVO & MODO & AMPX & 06/08/01 & t102 & \\
\hline 278 & 38086 & $\operatorname{sr} 86$ & 3831 & 38086 & ENDFB & V6 & RELO & REVO & MODO & AMPX & 06/08/01 & t102 & \\
\hline 279 & 38087 & $\operatorname{sr} 87$ & 3834 & 38087 & ENDFB & V6 & RELO & REVO & MODO & AMPX & 06/08/01 & t102 & \\
\hline 280 & 38088 & $\operatorname{sr} 88$ & 3837 & 38088 & ENDFB & V6 & RELO & REVO & MODO & AMPX & $06 / 08 / 01$ & t102 & \\
\hline 281 & 38089 & $\operatorname{sr} 89$ & 3840 & 38089 & ENDFB & V6 & RELO & REVO & MODO & AMPX & $06 / 08 / 01$ & t102 & \\
\hline 282 & 38090 & $\operatorname{sr} 90$ & 3843 & 38090 & ENDFB & V6 & RELO & REVO & MODO & AMPX & 06/08/01 & t102 & \\
\hline 283 & 1003 & t3 & 131 & 1003 & ENDFB & V6 & RELO & REVO & MODO & AMPX & $06 / 08 / 01$ & t101 & \\
\hline 284 & 73181 & ta181 & 7328 & 73181 & ENDFB & V6 & RELO & REVO & MODO & AMPX & $06 / 08 / 01$ & t106 & \\
\hline 285 & 73182 & ta182 & 7331 & 73182 & ENDFB & V6 & RELO & REVO & MODO & AMPX & $06 / 08 / 01$ & t106 & \\
\hline 286 & 65159 & tb159 & 6525 & 65159 & ENDFB & V6 & RELO & REVO & MODO & AMPX & $06 / 08 / 01$ & t106 & \\
\hline 287 & 65160 & tb160 & 6528 & 65160 & ENDFB & V6 & RELO & REVO & MODO & AMPX & $06 / 08 / 01$ & t106 & \\
\hline 288 & 43099 & tc99 & 4325 & 43099 & ENDFB & V6 & RELO & REVO & MODO & AMPX & $06 / 08 / 01$ & t104 & \\
\hline 289 & 52120 & te120 & 5225 & 52120 & ENDFB & V6 & RELO & REVO & MODO & AMPX & $06 / 08 / 01$ & t105 & \\
\hline 290 & 52122 & te122 & 5231 & 52122 & ENDFB & V6 & RELO & REVO & MODO & AMPX & $06 / 08 / 01$ & t105 & \\
\hline 291 & 52123 & te123 & 5234 & 52123 & ENDFB & V6 & RELO & REVO & MODO & AMPX & $06 / 08 / 01$ & t105 & \\
\hline 292 & 52124 & te124 & 5237 & 52124 & ENDFB & V6 & RELO & REVO & MODO & AMPX & $06 / 08 / 01$ & t105 & \\
\hline 293 & 52125 & te125 & 5240 & 52125 & ENDFB & V6 & RELO & REVO & MODO & AMPX & $06 / 08 / 01$ & t105 & \\
\hline 294 & 52126 & te126 & 5243 & 52126 & ENDFB & V6 & RELO & REVO & MODO & AMPX & $06 / 08 / 01$ & t105 & \\
\hline 295 & 52601 & te $127 \mathrm{~m}$ & 5247 & 52601 & endfb & v6 & relo & rev0 & $\bmod 0$ & ampx & $08 / 06 / 01$ & t105 & \\
\hline 296 & 52128 & te128 & 5249 & 52128 & ENDFB & V6 & RELO & REVO & MODO & AMPX & 06/08/01 & t105 & \\
\hline 297 & 52611 & te $129 m$ & 5253 & 52611 & endfb & v6 & relo & rev0 & $\bmod 0$ & ampx & 08/06/01 & t105 & \\
\hline 298 & 52130 & te130 & 5255 & 52130 & ENDFB & V6 & RELO & REVO & MODO & AMPX & 06/08/01 & t105 & \\
\hline
\end{tabular}




\begin{tabular}{|c|c|c|c|c|c|c|c|c|c|c|c|c|c|}
\hline Dentry & identifier & \multicolumn{11}{|c|}{ t i t $1 \mathrm{e}$} & source \\
\hline 299 & 52132 & te132 & 5261 & 52132 & ENDFB & V6 & RELO & REVO & MOD0 & AMPX & $06 / 08 / 01$ & t105 & 25 \\
\hline 300 & 90230 & $\operatorname{th} 230$ & 9034 & 90230 & ENDFB & V6 & RELO & REVO & MOD0 & AMPX & $06 / 08 / 01$ & t110 & 30 \\
\hline 301 & 90232 & $\operatorname{th} 232$ & 9040 & 90232 & ENDFB & V6 & REL0 & REVO & MOD1 & AMPX & $06 / 08 / 01$ & t109 & 31 \\
\hline 302 & 22000 & ti & 2200 & 22000 & endfb & v6 & relo & rev0 & $\bmod 0$ & ampx & $08 / 06 / 01$ & t102 & 32 \\
\hline 303 & 22046 & ti46 & 2225 & 22046 & ENDFB & V6 & REL0 & REVO & MOD0 & AMPXII & INCOMPLETE & t102 & \\
\hline 304 & 22047 & ti47 & 2228 & 22047 & ENDFB & V6 & REL0 & REVO & MOD0 & AMPXII & INCOMPLETE & t102 & \\
\hline 305 & 22048 & $\operatorname{ti} 48$ & 2231 & 22048 & ENDFB & V6 & RELO & REVO & MOD0 & AMPXII & INCOMPLETE & t 102 & \\
\hline 306 & 22050 & ti50 & 2237 & 22050 & ENDFB & V6 & RELO & REVO & MOD0 & AMPXII & INCOMPLETE & t 102 & \\
\hline 307 & 92232 & u232 & 9219 & 92232 & ENDFB & V6 & RELO & REVO & MOD1 & AMPX & $06 / 08 / 01$ & t109 & \\
\hline 308 & 92233 & u233 & 9222 & 92233 & ENDFB & V6 & REL0 & REVO & MOD1 & AMPX & $06 / 08 / 01$ & t109 & \\
\hline 309 & 92234 & u234 & 9225 & 92234 & ENDFB & V6 & RELO & REVO & MOD1 & AMPX & $06 / 08 / 01$ & t109 & \\
\hline 310 & 92235 & u235 & 9228 & 92235 & ENDFB & V6 & RELO & REV5 & MOD6 & AMPX & $06 / 08 / 01$ & rev5/tape. 144 & \\
\hline 311 & 92236 & u236 & 9231 & 92236 & ENDFB & V6 & RELO & REVO & MOD1 & AMPX & $06 / 08 / 01$ & t108 & \\
\hline 312 & 92237 & u237 & 9234 & 92237 & ENDFB & V6 & RELO & REV1 & MOD2 & AMPX & $06 / 08 / 01$ & rev2/tape. 129 & \\
\hline 313 & 92238 & u238 & 9237 & 92238 & ENDFB & V6 & RELO & REV3 & MOD4 & AMPX & $06 / 08 / 01$ & rev5/tape. 142 & \\
\hline 314 & 23000 & $\mathrm{v}$ & 2300 & 23000 & endfb & v6 & relo & rev0 & $\operatorname{mod1}$ & ampx & $08 / 06 / 01$ & t103 & \\
\hline 315 & 74000 & W & 7400 & 74000 & endfb & v6 & relo & rev1 & $\bmod 2$ & ampx & $08 / 06 / 01$ & t120 & \\
\hline 316 & 74182 & w182 & 7431 & 74182 & ENDFB & V6 & RELO & REV1 & MOD1 & AMPX & $06 / 08 / 01$ & rev6/tape. 151 & \\
\hline 317 & 74183 & w183 & 7434 & 74183 & ENDFB & V6 & RELO & REV1 & MOD1 & AMPX & $06 / 08 / 01$ & rev6/tape. 151 & \\
\hline 318 & 74184 & w184 & 7437 & 74184 & ENDFB & V6 & RELO & REV1 & MOD1 & AMPX & $06 / 08 / 01$ & rev6/tape. 151 & \\
\hline 319 & 74186 & w186 & 7443 & 74186 & ENDFB & V6 & RELO & REV1 & MOD1 & AMPX & $06 / 08 / 01$ & rev6/tape. 151 & \\
\hline 320 & 54124 & xe124 & 5425 & 54124 & ENDFB & V6 & RELO & REVO & MOD0 & AMPX & $06 / 08 / 01$ & t105 & \\
\hline 321 & 54126 & xe126 & 5431 & 54126 & ENDFB & V6 & RELO & REVO & MOD0 & AMPX & $06 / 08 / 01$ & t105 & 1 \\
\hline 322 & 54128 & xe128 & 5437 & 54128 & ENDFB & V6 & REL0 & REVO & MOD0 & AMPX & $06 / 08 / 01$ & t105 & \\
\hline 323 & 54129 & xe129 & 5440 & 54129 & ENDFB & V6 & REL0 & REVO & MOD0 & AMPX & $06 / 08 / 01$ & t105 & 3 \\
\hline 324 & 54130 & xe130 & 5443 & 54130 & ENDFB & V6 & REL0 & REVO & MOD0 & AMPX & $06 / 08 / 01$ & t105 & \\
\hline 325 & 54131 & xe131 & 5446 & 54131 & ENDFB & V6 & REL0 & REVO & MOD0 & AMPX & $06 / 08 / 01$ & t105 & \\
\hline 326 & 54132 & xe132 & 5449 & 54132 & ENDFB & V6 & RELO & REV0 & MOD0 & AMPX & $06 / 08 / 01$ & t105 & \\
\hline 327 & 54133 & xe133 & 5452 & 54133 & ENDFB & V6 & REL0 & REVO & MOD0 & AMPX & $06 / 08 / 01$ & t105 & I \\
\hline 328 & 54134 & xe134 & 5455 & 54134 & ENDFB & V6 & RELO & REVO & MOD0 & AMPX & $06 / 08 / 01$ & t105 & \\
\hline 329 & 54135 & xe135 & 5458 & 54135 & ENDFB & V6 & REL0 & REVO & MOD0 & AMPX & $06 / 08 / 01$ & t105 & 9 \\
\hline 330 & 54136 & xe136 & 5461 & 54136 & ENDFB & V6 & RELO & REVO & MOD0 & AMPX & $06 / 08 / 01$ & t105 & 0 \\
\hline 331 & 39089 & y89 & 3925 & 39089 & ENDFB & V6 & RELO & REV1 & MOD2 & AMPX & $06 / 08 / 01$ & rev4/tape.137 & \\
\hline 332 & 39090 & y90 & 3928 & 39090 & ENDFB & V6 & RELO & REVO & MOD0 & AMPX & $06 / 08 / 01$ & t102 & \\
\hline 333 & 39091 & y91 & 3931 & 39091 & ENDFB & V6 & RELO & REVO & MOD0 & AMPX & $06 / 08 / 01$ & t102 & \\
\hline 334 & 40000 & zr & 4000 & 40000 & endfb & v6 & relo & rev1 & $\bmod 2$ & ampx & $08 / 06 / 01$ & t 120 & \\
\hline 335 & 40701 & zr_zrh & 58 & 40701 & endfb & v6 & relo & rev0 & $\operatorname{mod1}$ & ampx & $08 / 06 / 01$ & rev3/tape. 133 & \\
\hline 336 & 40090 & $\operatorname{zr} \overline{9} 0$ & 4025 & 40090 & ENDFB & V6 & RELO & REVO & MOD0 & AMPX & $06 / 08 / 01$ & t104 & \\
\hline 337 & 40091 & $\operatorname{zr} 91$ & 4028 & 40091 & ENDFB & V6 & REL0 & REVO & MOD0 & AMPX & $06 / 08 / 01$ & t104 & \\
\hline 338 & 40092 & $\operatorname{zrg} 2$ & 4031 & 40092 & ENDFB & V6 & RELO & REVO & MOD0 & AMPX & $06 / 08 / 01$ & t104 & \\
\hline 339 & 40093 & $\operatorname{zr93}$ & 4034 & 40093 & ENDFB & V6 & RELO & REVO & MOD0 & AMPX & $06 / 08 / 01$ & t104 & \\
\hline 340 & 40094 & $\operatorname{zr} 94$ & 4037 & 40094 & ENDFB & V6 & RELO & REVO & MOD0 & AMPX & $06 / 08 / 01$ & t104 & \\
\hline 341 & 40095 & zr95 & 4040 & 40095 & ENDFB & V6 & RELO & REVO & MOD0 & AMPX & $06 / 08 / 01$ & t104 & \\
\hline 342 & 40096 & zr96 & 4043 & 40096 & ENDFB & V6 & REL0 & REVO & MOD0 & AMPX & $06 / 08 / 01$ & t104 & \\
\hline
\end{tabular}




\section{APPENDIX C \\ EXAMPLE OUTPUT OF PROCESSING CRC BENCHMARKS WITH CRCREAD}

The following information has been obtained by processing the CRC benchmark model cr3i12a with the CRCREAD program. The first two columns (i.e., symbol and ZA number) of the output correspond to the 130 isotopes as specified by the YMP for the evaluation of temperature effects. The remaining columns identify whether the specific isotope is present in the model. If an isotope is present, CRCREAD identifies the location of the specific isotope in the MCNP model.

\begin{tabular}{|c|c|c|c|c|c|}
\hline Symbol & $\mathrm{ZA}$ & Not Present & Fuel & Structure & Both \\
\hline------ & ----- & ----------- & ---- & --------- & ---- \\
\hline ag107 & 47107 & & & $\mathrm{x}$ & \\
\hline $\begin{array}{l}\text { ag109 } \\
\text { al27 }\end{array}$ & $\begin{array}{l}47109 \\
13027\end{array}$ & & & $\mathrm{x}$ & $\mathrm{x}$ \\
\hline $\operatorname{am} 241$ & 95241 & & $\mathrm{x}$ & & \\
\hline am $242 m$ & 95242 & & $\mathrm{x}$ & & \\
\hline am243 & 95243 & & $\mathrm{x}$ & & \\
\hline as 75 & 33075 & & $\mathrm{x}$ & & \\
\hline b10 & 5010 & & & $\mathrm{x}$ & \\
\hline b11 & 5011 & & & $\mathrm{x}$ & \\
\hline ba138 & 56138 & & $\mathrm{x}$ & & \\
\hline be9 & 4009 & $\mathrm{x}$ & & & \\
\hline C & 6000 & & & $\mathrm{x}$ & \\
\hline $\mathrm{cd}$ & 48000 & & & $\mathrm{x}$ & \\
\hline $\mathrm{cm} 242$ & 96242 & & $\mathrm{x}$ & & \\
\hline $\mathrm{cm} 243$ & 96243 & & $\mathrm{x}$ & & \\
\hline $\mathrm{cm} 244$ & 96244 & & $\mathrm{x}$ & & \\
\hline $\mathrm{cm} 245$ & 96245 & & $\mathrm{x}$ & & \\
\hline $\mathrm{cm} 246$ & 96246 & & $\mathrm{x}$ & & \\
\hline $\mathrm{cm} 247$ & 96247 & $\mathrm{x}$ & & & \\
\hline $\mathrm{cm} 248$ & 96248 & & $\mathrm{x}$ & & \\
\hline $\operatorname{co5} 9$ & 27059 & & & $\mathrm{x}$ & \\
\hline $\operatorname{cr} 50$ & 24050 & & & $\mathrm{x}$ & \\
\hline $\operatorname{cr} 52$ & 24052 & & & $\mathrm{x}$ & \\
\hline $\operatorname{cr} 53$ & 24053 & & & $\mathrm{x}$ & \\
\hline $\operatorname{cr} 54$ & 24054 & & & $\mathrm{x}$ & \\
\hline cs133 & 55133 & & $\mathrm{x}$ & & \\
\hline $\operatorname{cs} 135$ & 55135 & & $\mathrm{x}$ & & \\
\hline cu63 & 29063 & & & $\mathrm{x}$ & \\
\hline cu65 & 29065 & & & $\mathrm{x}$ & \\
\hline eu151 & 63151 & & $\mathrm{x}$ & & \\
\hline eu152 & 63152 & & $\mathrm{x}$ & & \\
\hline eu153 & 63153 & & $\mathrm{x}$ & & \\
\hline eu154 & 63154 & & $\mathrm{x}$ & & \\
\hline eu155 & 63155 & & $\mathrm{x}$ & & \\
\hline fe54 & 26054 & & & $\mathrm{x}$ & \\
\hline fe56 & 26056 & & & $\mathrm{x}$ & \\
\hline fe57 & 26057 & & & $\mathrm{x}$ & \\
\hline fe58 & 26058 & & & $\mathrm{x}$ & \\
\hline $\operatorname{gd152}$ & 64152 & & $\mathrm{x}$ & & \\
\hline gd154 & 64154 & & $\mathrm{x}$ & & \\
\hline gd155 & 64155 & & $\mathrm{x}$ & & \\
\hline gd156 & 64156 & & $\mathrm{x}$ & & \\
\hline
\end{tabular}




\begin{tabular}{|c|c|c|c|c|c|}
\hline Symbol & ZA & Not Present & Fuel & Structure & Both \\
\hline------ & ----- & ----------- & ---- & --------- & ---- \\
\hline gd157 & 64157 & & $\mathrm{x}$ & & \\
\hline gd158 & 64158 & & $\mathrm{x}$ & & \\
\hline gd160 & 64160 & & $\mathrm{x}$ & & \\
\hline h1 & 1001 & & & $\mathrm{x}$ & \\
\hline he4 & 2004 & & $\mathrm{x}$ & & \\
\hline ho165 & 67165 & & $\mathrm{x}$ & & \\
\hline in & 49000 & & & $\mathrm{x}$ & \\
\hline $\mathrm{kr} 80$ & 36080 & & $\mathrm{x}$ & & \\
\hline $\mathrm{kr} 82$ & 36082 & & $\mathrm{x}$ & & \\
\hline $\mathrm{kr} 83$ & 36083 & & $\mathrm{x}$ & & \\
\hline $\mathrm{kr} 84$ & 36084 & & $\mathrm{x}$ & & \\
\hline $\mathrm{kr} 86$ & 36086 & & $\mathrm{x}$ & & \\
\hline $1 i 6$ & 3006 & & $\mathrm{x}$ & & \\
\hline $1 i 7$ & 3007 & $\mathrm{x}$ & & & \\
\hline $\operatorname{mn} 55$ & 25055 & & & $\mathrm{x}$ & \\
\hline mo & 42000 & & & $\mathrm{x}$ & \\
\hline $\operatorname{mog} 5$ & 42095 & & $\mathrm{x}$ & & \\
\hline n14 & 7014 & & & $x$ & \\
\hline nb93 & 41093 & & & $\mathrm{x}$ & \\
\hline nd143 & 60143 & & $\mathrm{x}$ & & \\
\hline nd145 & 60145 & & $\mathrm{x}$ & & \\
\hline nd147 & 60147 & & $\mathrm{x}$ & & \\
\hline nd148 & 60148 & & $\mathrm{x}$ & & \\
\hline ni58 & 28058 & & & $x$ & \\
\hline ni 60 & 28060 & & & $\mathrm{x}$ & \\
\hline ni61 & 28061 & & & $\mathrm{x}$ & \\
\hline ni 62 & 28062 & & & $\mathrm{x}$ & \\
\hline ni64 & 28064 & & & $\mathrm{x}$ & \\
\hline np237 & 93237 & & $\mathrm{x}$ & & \\
\hline np238 & 93238 & & $\mathrm{x}$ & & \\
\hline 016 & 8016 & & & & $\mathrm{x}$ \\
\hline p31 & 15031 & & & $\mathrm{x}$ & \\
\hline pa233 & 91233 & $x$ & & & \\
\hline pd10 05 & 46105 & & $\mathrm{x}$ & & \\
\hline pa10 08 & 46108 & & $\mathrm{x}$ & & \\
\hline pm147 & 61147 & & $\mathrm{x}$ & & \\
\hline pm148 & 61148 & & $\mathrm{x}$ & & \\
\hline pm149 & 61149 & & $\mathrm{x}$ & & \\
\hline pr141 & 59141 & & $\mathrm{x}$ & & \\
\hline pu237 & 94237 & $\mathrm{x}$ & & & \\
\hline pu238 & 94238 & & $\mathrm{x}$ & & \\
\hline pu239 & 94239 & & $\mathrm{x}$ & & \\
\hline pu2 40 & 94240 & & $\mathrm{x}$ & & \\
\hline pu2 41 & 94241 & & $\mathrm{x}$ & & \\
\hline pu2 42 & 94242 & & $\mathrm{x}$ & & \\
\hline rh103 & 45103 & & $\mathrm{x}$ & & \\
\hline rh105 & 45105 & & $\mathrm{x}$ & & \\
\hline ru101 & 44101 & & $\mathrm{x}$ & & \\
\hline ru103 & 44103 & & $\mathrm{x}$ & & \\
\hline s32 & 16032 & & & $\mathrm{x}$ & \\
\hline si & 14000 & & & $\mathrm{x}$ & \\
\hline $\operatorname{sm} 147$ & 62147 & & $\mathrm{x}$ & & \\
\hline sm149 & 62149 & & $\mathrm{x}$ & & \\
\hline $\operatorname{sm} 150$ & 62150 & & $\mathrm{x}$ & & \\
\hline sm151 & 62151 & & $\mathrm{x}$ & & \\
\hline
\end{tabular}




\begin{tabular}{|c|c|c|c|c|}
\hline Symbol & ZA & Not Present & Fuel & Structure \\
\hline------ & ---- & ----------- & ---- & --------- \\
\hline sm152 & 62152 & & $\mathrm{x}$ & \\
\hline sn & 50000 & & & $\mathrm{x}$ \\
\hline $\operatorname{sn} 112$ & 50112 & $\mathrm{x}$ & & \\
\hline $\operatorname{sn} 114$ & 50114 & $\mathrm{x}$ & & \\
\hline $\operatorname{sn} 115$ & 50115 & $\mathrm{x}$ & & \\
\hline $\operatorname{sn} 116$ & 50116 & $\mathrm{x}$ & & \\
\hline $\operatorname{sn} 117$ & 50117 & $\mathrm{x}$ & & \\
\hline $\operatorname{sn} 118$ & 50118 & $\mathrm{x}$ & & \\
\hline $\operatorname{sn} 119$ & 50119 & $\mathrm{x}$ & & \\
\hline $\operatorname{sn} 120$ & 50120 & $\mathrm{x}$ & & \\
\hline $\operatorname{sn} 122$ & 50122 & $\mathrm{x}$ & & \\
\hline $\operatorname{sn} 123$ & 50123 & $\mathrm{x}$ & & \\
\hline $\operatorname{sn} 124$ & 50124 & $\mathrm{x}$ & & \\
\hline $\operatorname{sn} 125$ & 50125 & $\mathrm{x}$ & & \\
\hline $\operatorname{sn} 126$ & 50126 & $\mathrm{x}$ & & \\
\hline t3 & 1003 & & $\mathrm{x}$ & \\
\hline ta181 & 73181 & & & $\mathrm{x}$ \\
\hline tc99 & 43099 & & $\mathrm{x}$ & \\
\hline $\operatorname{th} 232$ & 90232 & $\mathrm{x}$ & & \\
\hline ti & 22000 & & & $\mathrm{x}$ \\
\hline u233 & 92233 & & $\mathrm{x}$ & \\
\hline u234 & 92234 & & $\mathrm{x}$ & \\
\hline u235 & 92235 & & $\mathrm{x}$ & \\
\hline u236 & 92236 & & $\mathrm{x}$ & \\
\hline u237 & 92237 & & $\mathrm{x}$ & \\
\hline u238 & 92238 & & $\mathrm{x}$ & \\
\hline xe131 & 54131 & & $\mathrm{x}$ & \\
\hline xe134 & 54134 & & $\mathrm{x}$ & \\
\hline xe135 & 54135 & & $\mathrm{x}$ & \\
\hline y89 & 39089 & & $\mathrm{x}$ & \\
\hline zr & 40000 & & & $\mathrm{x}$ \\
\hline $\operatorname{zr93}$ & 40093 & & $\mathrm{x}$ & \\
\hline
\end{tabular}




\section{INTERNAL DISTRIBUTION}

1. M. D. DeHart, 6011, MS-6370

2. M. E. Dunn, 6011, MS-6370

3. J. N. Herndon, 7601, MS-6305

4. C. V. Parks, 6011, MS-6370

5. R. M. Westfall, 6011, MS-6370

6. ORNL Laboratory Records, $4500 \mathrm{~N}$, MS-6254

\section{EXTERNAL DISTRUBITION}

7. D. Brownson, Bechtel SAIC Co., Inc., 1261 Town Center Drive, Las Vegas, NV 89144

8. T. Doering, Bechtel SAIC Co., Inc., 1261 Town Center Drive, Las Vegas, NV 89144

9. J. Scaglione, Bechtel SAIC Co., Inc., 1261 Town Center Drive, Las Vegas, NV 89144

10. D. A. Thomas, Bechtel SAIC Co., Inc., 1261 Town Center Drive, Las Vegas, NV 89144 